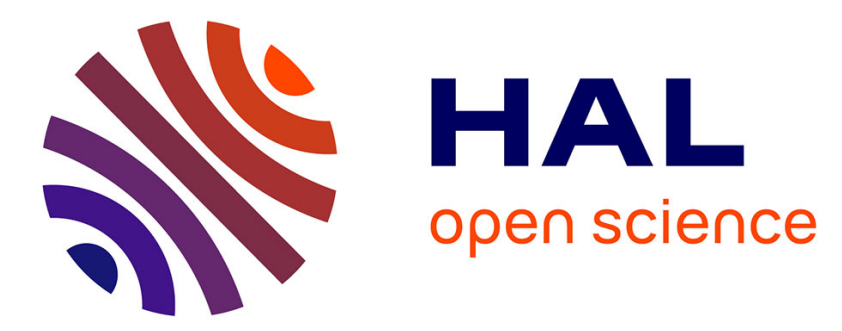

\title{
Numerical experiments of island stabilization by RF heating with stiff temperature profile
}

Patrick Maget, Fabien Widmer, Olivier Février, Hinrich Lütjens, Xavier

Garbet

\section{- To cite this version:}

Patrick Maget, Fabien Widmer, Olivier Février, Hinrich Lütjens, Xavier Garbet. Numerical experiments of island stabilization by RF heating with stiff temperature profile. Plasma Physics and Controlled Fusion, 2018, 60, pp.095003. 10.1088/1361-6587/aacf64 . cea-01871084

HAL Id: cea-01871084 https://hal-cea.archives-ouvertes.fr/cea-01871084

Submitted on 10 Sep 2018

HAL is a multi-disciplinary open access archive for the deposit and dissemination of scientific research documents, whether they are published or not. The documents may come from teaching and research institutions in France or abroad, or from public or private research centers.
L'archive ouverte pluridisciplinaire $\mathbf{H A L}$, est destinée au dépôt et à la diffusion de documents scientifiques de niveau recherche, publiés ou non, émanant des établissements d'enseignement et de recherche français ou étrangers, des laboratoires publics ou privés. 


\title{
Numerical experiments of island stabilization by RF heating with stiff temperature profile
}

\author{
Patrick Maget, Fabien Widmer, Olivier Février ${ }^{1}$, Hinrich \\ Lütjens $^{2}$, Xavier Garbet \\ CEA, IRFM, F-13108 Saint Paul-lez-Durance, France. \\ ${ }^{1}$ Swiss Plasma Center, Ecole Polytechnique Fédérale de Lausanne, CH-1015 \\ Lausanne, Switzerland. \\ ${ }^{2}$ Centre de Physique Théorique, Ecole Polytechnique, CNRS, France. \\ E-mail: patrick.maget@cea.fr
}

\begin{abstract}
The nature of turbulent transport in tokamak plasmas results in temperature profiles that are called resilient or stiff, and the stabilization of magnetic islands by a localized heat source is expected to be extremely sensitive to the stiffness strength. Theoretical expectations are verified with nonlinear simulations, showing a good agreement and confirming the enhanced stabilization efficiency due to large profile stiffness when the power used for the control is small compared with the heating power producing the equilibrium profiles. Heat sources that are present in the island region before the RF heating is applied contribute to reduce the island size, but at the same time, they severely damp the control capability.
\end{abstract}

\section{Introduction}

The growth of magnetic islands in tokamak plasmas results in a degradation of the energy confinement time that could be incompatible with the realization of a viable fusion reactor. In order to mitigate the associated risk, control methods based on the generation of a current opposing the island current have been successfully developed. The most common tools for achieving this control is the coupling of RF waves at the Electron Cyclotron (EC) frequency with the electron population, the interaction being sufficiently well focused to allow a localized action at the island O-point. This interaction produces a heating of the electron population, and, depending on the orientation of the EC beam, a localized current of variable amplitude. This technique has shown its efficiency in a large number of experiments [Maraschek, 2012], and an EC system is planned for the safe operation of ITER. The stabilization of an island by the EC driven current is generally more efficient than the heating [Lazzari and Westerhof, 2009, Lazzari and Westerhof, 2010]. However, the contribution of the later is not negligible and has been verified experimentally [Westerhof et al., 2007]. The analytical derivation of the island decay due to a heating source has been done originally for a non-stiff plasma, i.e. with a uniform diffusivity [Kurita et al., 1994, Hegna and Callen, 1997, Lazzari and Westerhof, 2009]. How- 
ever, numerical works [Hornsby et al., 2010, Hornsby et al., 2011, Zarzoso et al., 2015, Hill et al., 2015, Izacard et al., 2016, Navarro et al., 2017] and experimental analyzes [Inagaki et al., 2004, Ida et al., 2012, Bardòczi et al., 2016, Bardòczi et al., 2017] have shown that heat diffusivity is far from being uniform in the vicinity of an island. It is strongly decreased inside the separatrix, and slightly increased just outside. This can be explained by the turbulent heat transport properties that lead, in normal plasma conditions, i.e. in the absence of any Magneto-Hydro-Dynamic (MHD) activity, to resilient or stiff temperature profiles. The implications of profile stiffness for the island saturation are moderate when the island size is far above the characteristic transport width [Fitzpatrick, 2017]. However, the impact on the stabilization by a local heat source is expected to be large [Maget et al., 2018], and the purpose of the present work is to verify it numerically.

In the present paper, we therefore report on numerical simulations of island stabilization by a localized RF heat source, in a plasma with a stiff temperature profile. For this study, we use the nonlinear MHD code XTOR [Lütjens and Luciani, 2010] with the resistive MHD model, where additional equations covering the effect of a three dimensional RF source are implemented [Février et al., 2016, Février et al., 2018].The island decay rate that is obtained when the heat source is localized at the O-point of the island is in good agreement with theoretical expectations derived in similar conditions, i.e. for an island that is sufficiently large so that the temperature profile is flat before the RF application. We also evaluate numerically the stabilization potential for a continuous $\mathrm{RF}$ heat deposition in a plasma with a rotating island.

The paper is organized as follows: the MHD equations, transport model and the plasma equilibrium that is considered are presented in section 2. In section 3, we report on the settings and results of numerical simulations. The response of the plasma to the RF heating is first investigated, showing a strong localization at the deposition radius when stiffness is taken into account. The comparison with theoretical predictions derived in [Maget et al., 2018] shows a good agreement, and the background heating in the island region is shown to play against the control capability of the RF source when the stiffness is large. A simple criteria for anticipating this deleterious influence is the presence of a bump of temperature inside the island before the RF application. Finally, the role of the profile stiffness on the stabilization efficiency of a one dimensional source, that mimics that of a continuous RF deposition for a rotating island, is investigated.

\section{MHD model, Equilibrium, and simulation parameters}

\subsection{MHD model}

In the present work we consider the resistive MHD model:

$$
\begin{aligned}
& \left(\partial_{t}+\mathbf{V} \cdot \nabla\right) \rho=-\rho \nabla \cdot \mathbf{V}-\nabla \cdot \Gamma_{a n}+\Sigma \\
& \left(\partial_{t}+\mathbf{V} \cdot \nabla\right) p=-\Gamma p \nabla \cdot \mathbf{V}+H_{e q}+H_{R F}-(\Gamma-1) \nabla \cdot \mathbf{q}_{\chi} \\
& \rho\left(\partial_{t}+\mathbf{V} \cdot \nabla\right) \mathbf{V}=\mathbf{J} \times \mathbf{B}-\nabla p+\nabla \cdot \nu \nabla \mathbf{V}
\end{aligned}
$$


$\partial_{t} \mathbf{B} \quad=-\nabla \times \mathbf{E}$

with $\rho$ the mass density, $p$ the total pressure, $\mathbf{V}=\mathbf{V}_{\mathbf{E}}+\mathbf{V}_{\| \mathbf{i}}, \mathbf{V}_{\mathbf{E}}=\mathbf{E} \times \mathbf{B} / B^{2}$ and $\mathbf{V}_{\| \mathbf{i}}$ the parallel ion velocity. The ratio of specific heat coefficient is $\Gamma=5 / 3$, $H_{e q} \equiv-(\Gamma-1) \nabla \cdot \chi_{\perp} \nabla_{\perp} p_{e q}$ is the heat source $\left(p_{e q} \equiv p(t=0)\right), H_{R F}$ is the RF heat source and $\mathbf{q}_{\chi}=-\rho \chi_{\|} \mathbf{b}(\mathbf{b} \cdot \nabla T)-\rho \chi_{\perp} \nabla_{\perp} T$ is the diffusive heat flux $(\mathbf{b} \equiv \mathbf{B} / B)$, with $T=p / \rho$. The turbulent particle flux is composed of a diffusive and a convective part, $\Gamma_{a n}=-D_{\perp} \nabla \rho+\rho \mathbf{V}_{\text {pinch }}$, where the pinch velocity is defined so that there is no particle source $(\Sigma)$ inside the radial position $\sqrt{\psi}=0.9$, as described in [Maget et al., 2016].

The Ohm's law is given by

$$
\mathbf{E}+\mathbf{V} \times \mathbf{B}=\eta\left[\mathbf{J}-\mathbf{J}_{\mathbf{C D}}\right]
$$

with $\mathbf{J}_{\mathbf{C D}}$ the non-inductive current density source. We choose the current to have a large inductive part $\mathbf{J}_{\mathbf{C D}}=\left(\mathbf{J}_{\varphi}-E_{0} / \eta\right)_{t=0}$ with $E_{0}$ a constant prescribed at the edge such that $E_{0} /\left(\eta(0) J_{\varphi}(0)\right)=1$, i.e. the current is fully inductive at the plasma center [Février et al., 2018].

The RF heat source is implemented as follows [Février et al., 2016]:

$$
\begin{aligned}
& \partial_{t} H_{R F}=\nu_{f}\left(H_{R F}^{S}-H_{R F}\right)-\nabla \cdot \mathbf{q}_{\mathbf{R F}}^{\mathbf{H}} \\
& \mathbf{q}_{\mathbf{R F}}^{\mathbf{H}}=-\chi_{\perp}^{R F} \nabla H_{R F}-\left(\chi_{\|}^{R F}-\chi_{\perp}^{R F}\right) \mathbf{b b} \cdot \nabla H_{R F}
\end{aligned}
$$

where $\nu_{f}=\nu_{e i}\left(\mathrm{v}_{t h} / \mathrm{v}_{\text {res }}\right)^{3}$ is the collision frequency of the fast electrons. In the following we take $\mathrm{v}_{t h} / \mathrm{v}_{\text {res }}=1 / 2$.

The source term $H_{R F}^{S}$ are defined as 1D or 3D Gaussian:

$H_{R F}^{S}(y, \theta, \phi)=H_{0}^{S} \exp \left(-\frac{\left(y-y_{R F}^{0}\right)^{2}}{2 \sigma_{r}^{2}}-\frac{\left(\theta-\theta_{R F}^{0}\right)^{2}}{2 \sigma_{\theta}^{2}}-\frac{\left(\phi-\phi_{R F}^{0}\right)^{2}}{2 \sigma_{\phi}^{2}}\right)$

with $y \equiv \sqrt{\psi}$. The source width $\delta_{R F}$ is defined as the radial full width at half maximum of the source, and is given by $\delta_{R F}=2 \sqrt{2 \ln 2} \sigma_{r}$.

\subsection{Anomalous transport model}

Heat and particle transport in tokamaks are generally dominated by turbulent processes that are triggered above some critical gradient, positive or negative [Romanelli et al., 2004]. This has been well documented in theoretical works, in the linear and nonlinear regimes [Rebut et al., 1988, Dimits et al., 2000, Garbet et al., 2004], and has also received several experimental confirmations [Imbeaux et al., 2001, Mantica et al., 2009, DeBoo et al., 2012]. This issue is of interest not only for the understanding of the energy confinement time, but also in the context of magnetic island studies, since the flattening of temperature profile inside an island is expected to reduce locally the turbulent transport [Hornsby et al., 2010, Hornsby et al., 2011, Zarzoso et al., 2015, Hill et al., 2015, Izacard et al., 2016, Navarro et al., 2017]. This damping of turbulent processes at the O-point of magnetic island has also been deduced from experimental observations in stellarators [Inagaki et al., 2004] as well as in tokamaks [Ida et al., 2012, Bardòczi et al., 2017]. This distribution of turbulent diffusivity 
impacts the nonlinear evolution of a magnetic island, especially when its width competes with the characteristic width that equilibrates parallel and perpendicular fluxes outside the island, leading to a faster growth [Fitzpatrick, 2017].

In the context of island stabilization by RF heating, the level of turbulent transport inside the island is also a key parameter. Indeed, when part of the plasma current is sustained by a parallel electric field (the ohmic current), lowering plasma resistivity at the O-point helps forming a current filament that opposes that of the island [Hegna and Callen, 1997]. In this context, a low transport will allow the temperature to rise to higher value when a localized heat source is focused at the O-point, thus enhancing the control capability.

The dependence of turbulent transport on the temperature gradient is modeled as follows [Maget et al., 2018]:

$$
\chi_{\perp}=\chi_{\perp}^{r e f}\left|T^{\prime} / T_{e q}^{\prime}\right|^{\sigma-1}
$$

where the prime refers to the derivative in the radial direction, $T_{e q}$ is the initial equilibrium temperature and $\sigma$ is the stiffness parameter. This model ensures that the equilibrium temperature gradient is consistent with the input power $\left(H_{e q}\right.$ in the pressure equation), and provides the desired behavior leading to a strong excitation of turbulent transport when the stiffness parameter $\sigma$ is large. The level of heat transport exhibits a smooth transition at around $\left|T_{c r i t}^{\prime} / T_{e q}^{\prime}\right|=1-1 /(\sigma-1)$ between a low transport regime representative of collisional processes, and a high transport regime representative of turbulent ones (see figure 1). With this description, the plasma is in the turbulent regime everywhere in its initial equilibrium configuration.

In order to avoid too high and too low diffusivity, both being physically unrealistic and computationally difficult, we apply to the ratios $y=\left(\left|T^{\prime} / T_{e q}^{\prime}\right|, \chi_{\perp} / \chi_{\perp}^{r e f}\right)$ the following regularization :

$$
y_{\text {limited }}=\frac{G^{-1}+(1+g)^{-1}}{G^{-1}+(y+g)^{-1}}
$$

with $G$ and $g$ the maximum and minimum ratio that are allowed : the diffusivity can be at maximum $G$ times higher that its equilibrium ("ref") value, and at minimum $g$ times smaller. The lower value can be interpreted as the remaining collisional (i.e. neoclassical) transport when turbulent modes are stable. In the following, we take $G=1 / g=100$, which corresponds to a very low level of collisional transport.

We also mention for completeness that the perpendicular diffusivity coefficients $\left(D_{\perp}^{r e f}, \chi_{\perp}^{r e f}\right)$ are dependent on temperature following the gyro-Bohm scaling (i.e. proportional to $T^{3 / 2}$ ), and are formally related with plasma resistivity in the code as $\left(D_{\perp}^{r e f}, \chi_{\perp}^{r e f}\right)=\left(D_{\perp}^{0}, \chi_{\perp}^{0}\right)\left(\eta_{e q} / \eta_{0}\right)\left(\eta_{e q} / \eta\right)$, with $\eta_{e q}$ the initial (equilibrium) resistivity profile, $\eta_{0} \equiv \eta_{e q}(0),\left(D_{\perp}^{0}, \chi_{\perp}^{0}\right)$ the central values of diffusion and diffusivity coefficients, and $\eta$ the actual value of plasma resistivity that varies as $T^{-3 / 2}$. 


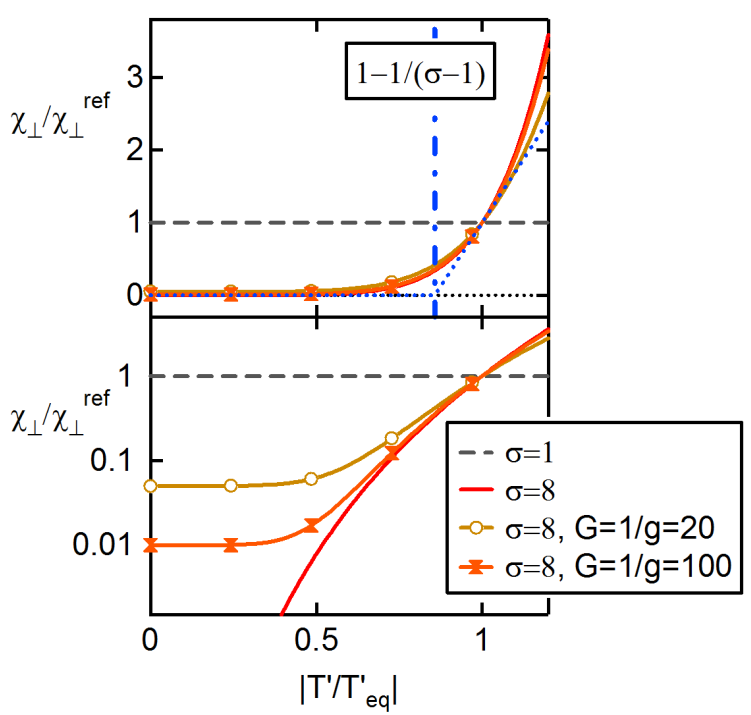

Figure 1. Perpendicular diffusivity normalized to its reference value, as a function of the temperature gradient relative to its equilibrium value, for stiffness parameters $\sigma=1$ and $\sigma=8$, and with the regularizations $G=1 / g=20$ and 100. Top: linear scale, bottom: logarithmic scale.

\subsection{Rutherford equation associated with the stiffness model}

The stiffness model presented above can be applied to derive an evolution equation for the island width under the effect of a localized heat source at the O-point. For a large island, such that the temperature is completely flattened inside the island separatrix, the associated Rutherford equation [Rutherford, 1973] is of the form [Maget et al., 2018]:

$$
I_{1} \tau_{R} \partial_{t} W=a \Delta^{\prime}+a \Delta_{\Omega}^{\prime}\left(P_{R F}\right)
$$

with $W \equiv w / a$ the island width normalized to the minor radius $a$ of the plasma, $\tau_{R}=$ $\mu_{0} a^{2} / \eta$ the resistive time, $I_{1} \approx 0.82, \Delta^{\prime}$ the tearing stability index [Furth et al., 1963], and

$$
a \Delta_{\Omega}^{\prime}\left(P_{R F}\right)=-C_{\Omega}\left(\mu_{c}, \sigma\right) \frac{a}{\mathcal{J}} \frac{q}{s} \frac{\mu_{0} R J_{\Omega}}{B_{z}} \frac{P_{e q}}{N \chi_{\perp}^{r e f} T_{s}}\left(\frac{P_{R F}}{P_{e q}}\right)^{1 / \sigma}
$$

with

$C_{\Omega}\left(\mu_{c}, \sigma\right) \approx \frac{3}{4 \pi^{2}}\left[0.8+\frac{0.6}{\sigma}-1.09 \frac{\mu_{c}}{\sigma}+0.24\left(\frac{\mu_{c}}{\sigma}\right)^{2}-0.23 \frac{\mu_{c}}{\sigma} \ln \frac{\mu_{c}}{\sigma}\right]$

where $\mathcal{J} \approx r R$ is the Jacobian and $J_{\Omega}$ the ohmic current at the resonance, $P_{R F}$ the total RF power injected at the O-point over the width $\delta_{H}, \mu_{c}=\left(\delta_{H} / W\right)^{2}, P_{e q}$ the power injected inside the resonant surface (in the absence of RF heating), $N$ the plasma density and $T_{s}$ the temperature at the resonance.

\subsection{Equilibrium}

We consider a magnetic equilibrium with circular cross-section at moderate beta in toroidal geometry, where the $(m=2, n=1)$ tearing mode, located at $\sqrt{\psi}=0.5$ with 
$\psi$ the normalized poloidal magnetic flux, is linearly unstable. The pressure profile is given by $\partial_{\psi} p \propto(1-\psi)$ and the current density profile by $I^{*} \propto(1-\psi)^{2}$ (see [Lütjens et al., 1996] for the definition of $\left.I^{*}\right)$. The inverse aspect ratio is $\epsilon \equiv a / R=0.3$ with $R$ and $a$ the major and minor radii of the torus, the normalized radius is defined as $r / a=x=\sqrt{\Phi}$ with $\Phi$ the normalized toroidal magnetic flux (the island is at $x \approx 0.34$ ), and the magnetic shear $s$ is about 0.585 at the island position. At magnetic axis $\left(R_{0}=2.4 \mathrm{~m}\right)$, the magnetic field is $B_{0}=3 T$, the ion density $n_{i}(0)=2 \times 10^{19} \mathrm{~m}^{-3}$, electron temperature $T_{e}(0)=3910 \mathrm{eV}$, and $\tau \equiv T_{i} / T_{e}=1$, these quantities being chosen so that they are consistent with the CHEASE equilibrium $: p(0)=e n_{i}(0) T_{e}(0)[1+\tau]$. As mentioned above, the plasma current density is fully inductive at the plasma center, but the condition that $\eta J_{\varphi}$ is equal to the same constant everywhere is not satisfied. The magnetic equilibrium is therefore maintained by a small non-inductive current $\left(\mathbf{J}_{\mathbf{C D}}\right)$ [Février et al., 2018]. At $q=2$, the inductive current density represents however about $83 \%$ of the total current density. The Alfvén time is $\tau_{A}=2.3 \times 10^{-7} \mathrm{~s}$, and the Lundquist number $\left(S=\tau_{R} / \tau_{A}\right)$ consistent with input parameters is $S_{0} \approx 80 \times 10^{7}$ at the plasma core. Since we will run simulations at $S_{0}=10^{7}$, parameters like viscosity $\nu$ and diffusion coefficients $\left(D_{\perp}, \chi_{\perp}\right)$ are rescaled such that the magnetic Prandtl number $\operatorname{Prm}=\left(\mu_{0} a^{2} \nu / \eta_{0}\right)$ and ratio of resistive to confinement times $\tau_{R} / \tau_{E}=\mu_{0} \chi_{\perp}^{0} / \eta_{0}=\left(\mu_{0} \chi_{\perp}^{0} / \eta_{0}\right)^{\text {real }}$ stay in a realistic range. We will take $\operatorname{Prm}=10$, $\mu_{0} \chi_{\perp}^{0} / \eta_{0}=150, D_{\perp}^{0}=2 / 3 \chi_{\perp}^{0}$ and $\chi_{\|} / \chi_{\perp}^{0}=10^{8}$ for the first part of the nonlinear simulation, and then $\chi_{\|} / \chi_{\perp}^{0}=4 \times 10^{8}$. The simulation time $t$ (normalized to the Alfvén time in the code) is converted into real time by computing $t[s]=t \times \tau_{A} \times S_{0} / S^{r e a l}$. The power that is injected inside the resonant surface position at $q=2$ is $P_{e q}=1.3 \mathrm{MW}$.

We will consider for our study two values of the stiffness parameter, $\sigma=1$ that corresponds to a uniform diffusivity, and $\sigma=8$ that corresponds to a realistic value of the stiffness expected for ITER [Kinsey et al., 2011].

\section{Numerical results}

Numerical simulations have been performed with a radial resolution of 512 points and a poloidal resolution of 96 points. The nonlinear regime has first been reached with $\sigma=1, \chi_{\|} / \chi_{\perp}^{0}=10^{8}$ and a toroidal resolution of 16 points (the toroidal mode numbers that are considered are $n=0, \cdots, n_{\max }$ with $\left.n_{\max }=4\right)$ and then 24 points $\left(n_{\max }=7\right)$. Note that the limitation in $n_{\max }$ comes from aliasing constraints [Press et al., 2007]. The characteristic transport width [Fitzpatrick, 1995] is then $W_{\chi}=2 \sqrt{2}\left(\chi_{\perp} / \chi_{\|}\right)^{1 / 4} \sqrt{x /(\epsilon n s)}=0.047$. The simulation have then been pursued (from $t=3.524 \mathrm{~s}$, see figure 2) with a larger $\chi_{\|}$in order to better flatten the temperature profile inside the island, a condition that is mandatory for a relevant comparison with the theoretical model. By taking $\chi_{\|} / \chi_{\perp}^{0}=4 \times 10^{8}$, we obtain $W_{\chi}=0.034$ and a flattening that covers most of the inner island region, as will be shown latter. This large value of

$\chi_{\|}$puts strong constraints on the simulation time step, that does not exceed few $10^{-2}$ Alfvén times, despite the simplicity of the MHD model (no diamagnetic rotations) that 

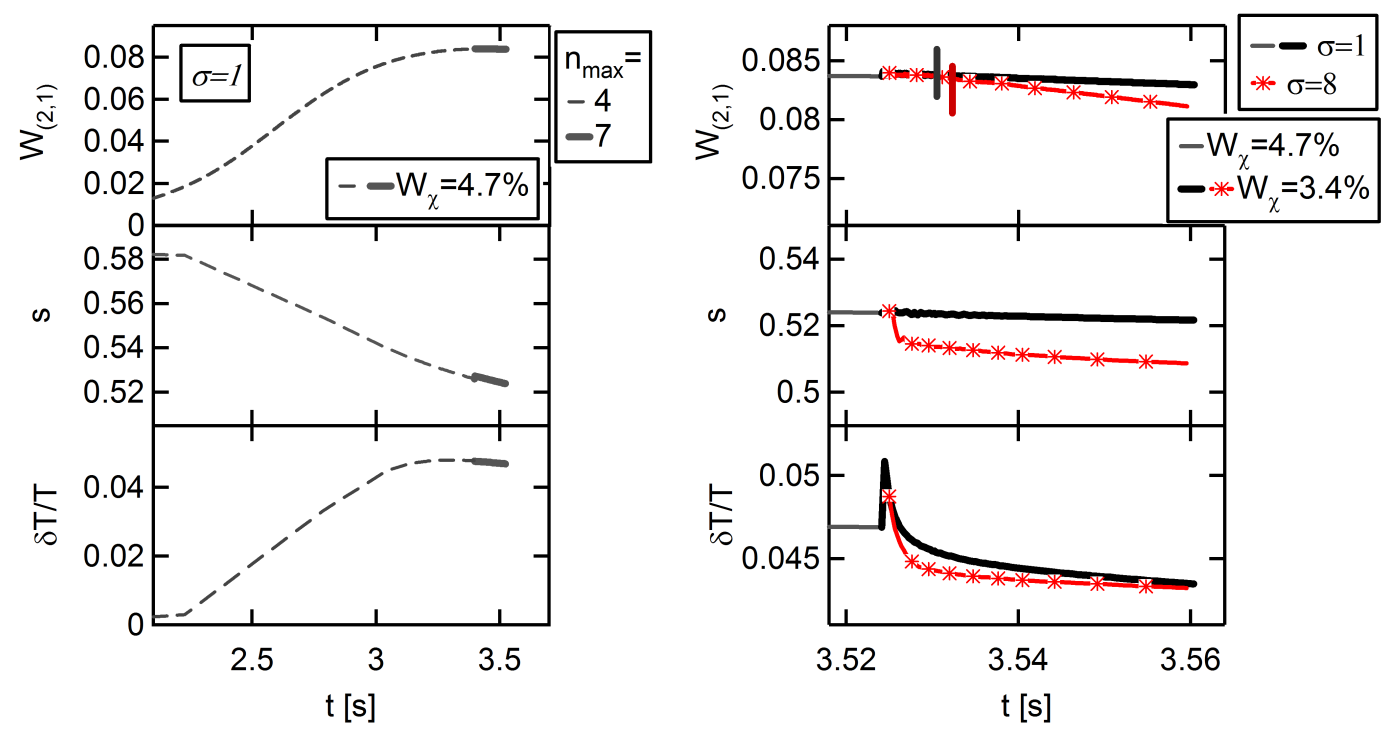

Figure 2. Saturation of the $(2,1)$ island: island width (top), magnetic shear at the resonance (middle) and relative temperature perturbation in the island region (bottom). Left: simulation with $\sigma=1, W_{\chi}=0.047$ and $n_{\max }=4$. Right: simulations with $\sigma=1$ and $\sigma=8, W_{\chi}=0.034$ and $n_{\max }=7$. Vertical bars indicate the time when RF heating is applied.

is used.

\subsection{Island saturation}

The $(2,1)$ island saturates at a width of about $8.4 \%$ for $\sigma=1$ and $W_{\chi}=0.047$, and its width decreases slowly when the parallel diffusivity is increased $\left(W_{\chi}=0.034\right)$, an effect that is consistent with a larger curvature stabilization [Lütjens et al., 2001] (see figure 2 ). In the figure, the magnetic shear is computed from the $n=0$ projection of the fields, and the relative temperature perturbation is defined as $\delta T / T=\max _{x}\left(T-T_{n=0}\right) / T_{n=0}$. The jump in $\delta T / T$ is due to the stronger contrast between inside/outside the island separatrix when $W_{\chi}$ is modified, a contrast that is shortly mediated by heat diffusivity. The decrease of the saturated island width is more pronounced for a stiffness parameter $\sigma=8$ (see section 3.2.4 for an explanation), but it is slow enough compared with the time scale of the RF stabilization not to perturb our study.

The profiles of temperature and perpendicular diffusivity at the O- and X-points of the $(2,1)$ island at saturation are shown in figure 3 for $\sigma=1$ and $\sigma=8$. In the later case, we observe the low level of transport inside the island, but we also see that outside the island separatrix (both at the $\mathrm{O}$ - and $\mathrm{X}$-points), the transport is impacted. In particular, we see that the transport level is increased outside the separatrix at the poloidal position of the O-point, as found in nonlinear gyrokinetic simulations [Navarro et al., 2017] and observed experimentally [Bardòczi et al., 2017]. The heat power that is present in the plasma to sustain the initial pressure profile is not completely negligible at the island location: in the region $\sqrt{\psi} \in[0.4,0.5]$, a power of $0.55 \mathrm{MW}$ is injected. The power 


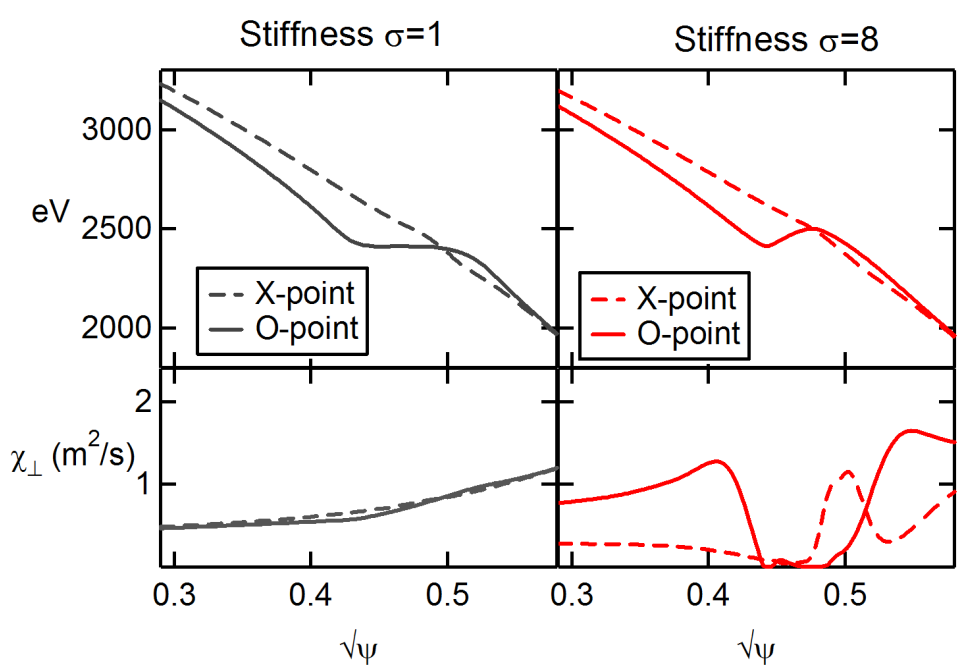

Figure 3. Temperature profile (top) and perpendicular diffusivity profile (bottom) at the X-and O-points of the saturated island at the time of the RF application, for a stiffness parameter $\sigma=1$ (left) and $\sigma=8$ (right), with the original background heat source (see figure 4 ).

injected inside the island can also be evaluated as a function of its size as

$$
P_{e q}^{i s l}=8 \pi w \mathcal{J} H_{e q}
$$

which gives for $H_{e q}(q=2) \approx 0.46 \mathrm{MW} / \mathrm{m}^{-3}$ and an island of full width $W=w / a \approx 8.4 \%$ a power of about $0.41 \mathrm{MW}$. This represents about $31 \%$ of the total power injected inside the resonant position $(\sqrt{\psi} \leq 0.5)$, i.e. $P_{e q}=1.3 M W$. This explains the bump of temperature inside the island for $\sigma=8$. Note that a similar bump has sometimes been observed experimentally [de Vries et al., 1997, Urso et al., 2010], and could be due as well to a residual heat source associated with a low diffusivity inside the island.

In order to be in conditions closer to the analytical case, we need to damp the heat source in the island region, and to compensate inside in order to keep the out-going flux at $q=2$ (i.e. $P_{e q}$ ) unchanged. This is obtained with a correction function :

$$
\begin{aligned}
H_{e q}^{\prime} & =H_{e q} \times \mathcal{F}(y) \\
\mathcal{F}(y) & =\left(1-f_{i s l}(y)\right)\left(1+\alpha f_{c}(y)\right) \\
f_{i s l}(y) & =\exp \left[-\left(\frac{y-y_{i}}{\sigma_{i}}\right)^{p}\right] \\
f_{c}(y) & =\exp \left[-\left(\frac{y}{\sigma_{c}}\right)^{q}\right]
\end{aligned}
$$

with $y \equiv \sqrt{\psi}, y_{i}=0.47, \sigma_{i}=0.04, p=8, \sigma_{c}=0.35, q=6, \alpha=0.85$. The original heat source and its modified version are shown in figure 4 . Now the power injected in the region $\sqrt{\psi} \in[0.4,0.5]$ is only of $0.16 \mathrm{MW}\left(12 \%\right.$ of $\left.P_{e q}\right)$, and in $\sqrt{\psi} \in[0.44,0.5]$ it is about $3 \mathrm{~kW}$ (about $0.3 \%$ of $P_{e q}$ ). 


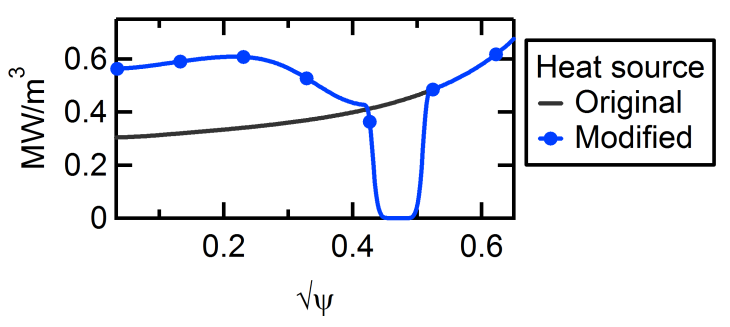

Figure 4. Heat source profile and its modified version (no heating in the island region).

The saturated island, after application of this modified heat source, evolves to a different state (figure 5). Due to the significant increase of the background heat source in the plasma core, the pressure increase is noticeable for $\sigma=1$, and very moderate, as expected, in the case $\sigma=8$. The large increase of the core pressure in the case $\sigma=1$ has a weak impact on the current profile for the time of the simulation, since we have a ratio $\tau_{R} / \tau_{E}=150$ that implies a slow evolution of the current profile compared to the pressure profile. However, this represents a transient phase, with a parallel electric field that is not flat radially, and a relative temperature fluctuation in the island region that is larger by more than $40 \%$ at the time when the RF heating is applied (vertical bars in figure 5). The situation for the modified heat source is therefore no longer similar to the original case, given the important role of the temperature fluctuation amplitude for the stabilization by localized heating [Maget et al., 2018], and the configuration ( $\sigma=1$, modified heat source) cannot be included in the comparison of the stiffness effect. Since the condition of flat temperature inside the island was already obtained for $\sigma=1$ with the original heat source, we conclude that the comparison with the analytical model is in fact more appropriate in this original case than for the modified heat source. The simulations have be performed however in the two cases, and we will see later that the larger contrast on the temperature fluctuation enhances the response to the RF heating, and leads to decay rates that are larger than the prediction of the analytical model.

In the case with $\sigma=8$, the temperature is now completely flattened at the O-point (figure 6), and the large stiffness prevents any side-effects like those observed with $\sigma=1$. The study is in this case as close as possible to the hypotheses of the analytical model.

\subsection{Island stabilization by localized RF heating}

We now apply a localized RF heat source at the O-point of the saturated island. Due to the absence of diamagnetic effects, the island does not rotate and the heat source stays at the O-point throughout the simulation. This situation is rarely encountered in experimental conditions, where diamagnetic rotations combined with the rotation of the plasma usually leads to rotating islands. The island rotation can be controlled by externally applied magnetic perturbations, as reported in [Westerhof et al., 2007, Volpe et al., 2009], but static islands generally end with a plasma disruption. However, 


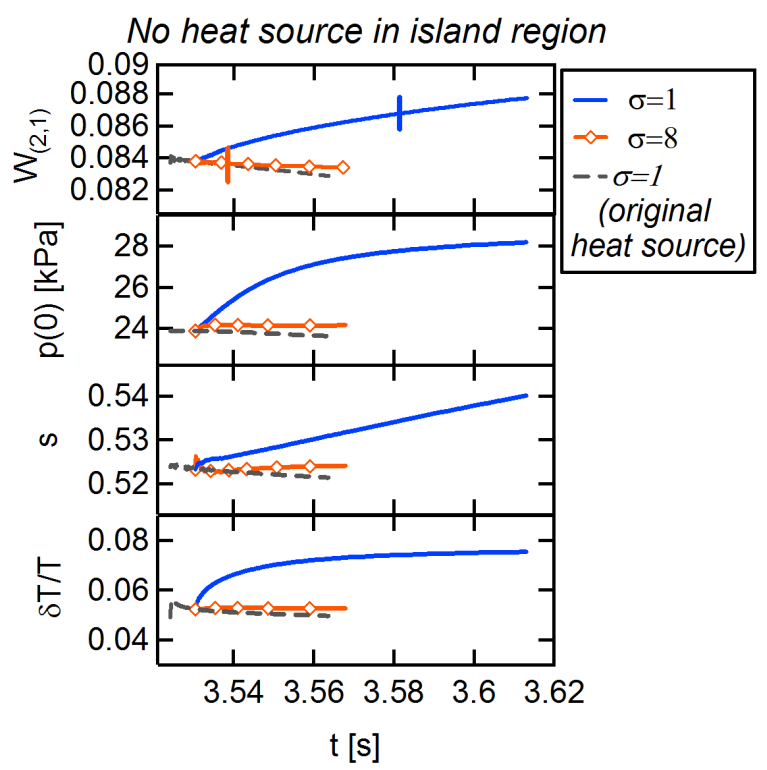

Figure 5. Saturation of the $(2,1)$ island for $\sigma=1$ and $\sigma=8$ in the alternative configuration without heat source in the island region: from top to bottom, island width, core pressure, magnetic shear at the resonance and relative temperature perturbation in the island region. Vertical bars indicate the time when RF heating is applied.

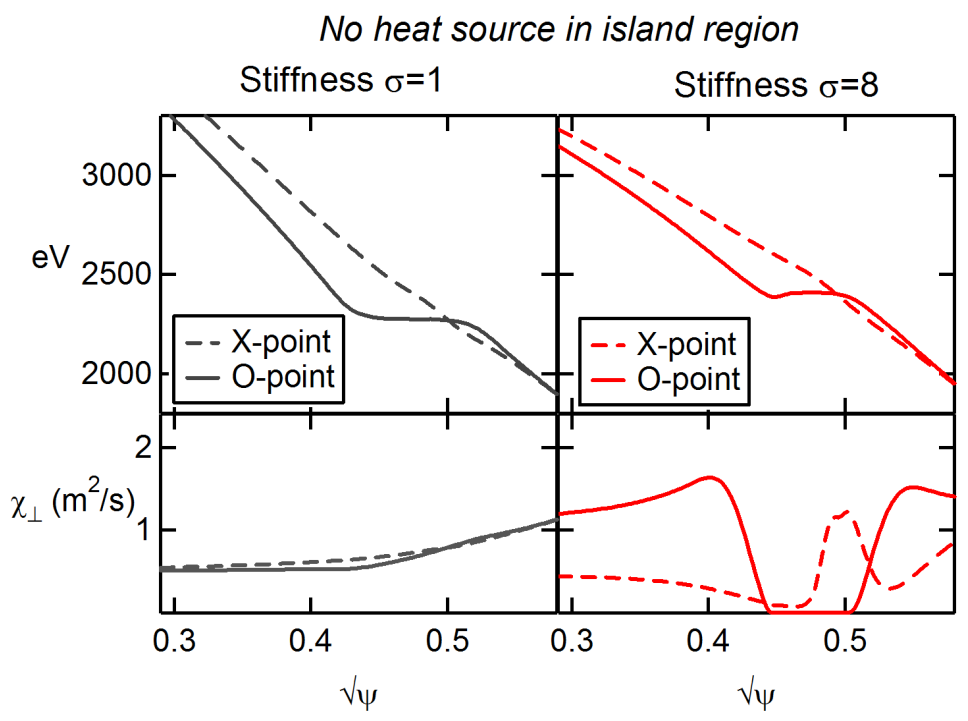

Figure 6. Temperature profile (top) and perpendicular diffusivity profile (bottom) at the $\mathrm{X}$ - and O-points of the saturated island at the time of the RF application, in the alternative configuration without heat source in the island region, for a stiffness parameter $\sigma=1$ (left) and $\sigma=8$ (right). 
the development of control techniques using two RF antennas has demonstrated the possibility to obtain a virtually continuous O-point deposition even when the island is rotating [Kasparek et al., 2016]. This experimental realization is equivalent to our simulation settings.

3.2.1. Settings A localized RF power source is applied at one O-point of the $(2,1)$ island, with the following specifications: $y_{R F}^{0}=0.48$ (the radial position of the O-point is slightly inside the resonant position because of the island asymmetry [Février et al., 2016]), $\theta_{R F}^{0}=\pi / 2$ (the O-point is at $\theta_{R F}^{0}= \pm \pi / 2$ ), $\varphi_{R F}^{0}=0$. In order to ensure a consistent deposition size in the radial, poloidal and toroidal directions, we should have in the case of a Heaviside deposition function of radial width $\delta_{H}$ : $\Delta \theta=(2 / m) \arccos \left(1-2 \mu_{c}\right)$ and $\Delta \varphi=(2 / n) \arccos \left(1-2 \mu_{c}\right)$, with $\mu_{c} \equiv\left(\delta_{H} / W\right)^{2}$. For Gaussian profiles (as we have in the simulations), we identify $\delta_{H}$ with $\delta_{R F}$, and we obtain the relations:

$$
\begin{aligned}
& \sigma_{\theta}=\frac{2}{m} \frac{\arccos \left(1-2 \mu_{c}\right)}{\delta_{H}} \sigma_{r}=\frac{\arccos \left(1-2 \mu_{c}\right)}{m \sqrt{2 \ln 2}} \\
& \sigma_{\varphi}=\frac{2}{n} \frac{\arccos \left(1-2 \mu_{c}\right)}{\delta_{H}} \sigma_{r}=\frac{\arccos \left(1-2 \mu_{c}\right)}{n \sqrt{2 \ln 2}}
\end{aligned}
$$

For an island width $W=8.4 \%, m=2, n=1$ and a source of width $\sigma_{r}=0.005$, we take $\sigma_{\theta}=0.12$ and $\sigma_{\varphi}=0.24$.

The radial width of the $\mathrm{RF}$ deposition, initially at $\delta_{R F} \approx 0.012$, is broadened by diffusion (see equation 6), and the effective RF deposition width is evaluated by computing the half-width of the RF power density at the O-point. This gives $\delta_{R F}^{e f f} \approx 0.037\left(\mu_{c} \approx 0.2\right)$, so that the heat source remains very localized inside the island. We take for the parallel RF diffusivity $\chi_{\|}^{R F}=\chi_{\|}$. The normalized contours of the power density source $H_{R F}^{S}$ and coupled power density $H_{R F}$ (10 ms after the RF power is switched on) are shown in figure 7 at the toroidal position $\varphi=0$. This shows the propagation of the heat source along field lines, concentrated at the O-point, and the broadening due to perpendicular transport. The heat source is equilibrated along the $q=2$ field line after a short characteristic time $\tau_{\|}=(R q)^{2} / \chi_{\|}^{R F} \approx 2 \times 10^{-7} s$, while the time scale for the rise of the $\mathrm{RF}$ heat source is $\tau_{f}=\nu_{f}^{-1} \approx 1 \mathrm{~ms}$.

The amplitude of the RF source is scanned in the range 0.1 to $1.8 \mathrm{MW}$, that is to say between $8 \%$ and $137 \%$ of the background power already injected inside $q=2\left(P_{e q}\right)$.

3.2.2. Prediction from the analytical model Theoretical predictions can be derived using the analytical model (see section 2.3). At $q=2$ we have $\mu_{0} R J_{\Omega} / B_{z} \approx 0.5524$, $T_{s}=2350 \mathrm{eV}, N \approx 1.838 \times 10^{-19} \mathrm{~m}^{-3}, \mathrm{~s}=0.585, \chi_{\perp}^{\text {ref }} \approx 0.908 \mathrm{~m}^{2} / \mathrm{s}, \mathrm{r} / \mathrm{a}=0.34$ and $P_{e q}=1.3 M W$, so that

$$
\frac{a}{\mathcal{J}} \frac{q}{s} \frac{\mu_{0} R J_{\Omega}}{B_{z}} \frac{P_{e q}}{N \chi_{\perp}^{r e f} T_{s}} \approx 476.8
$$




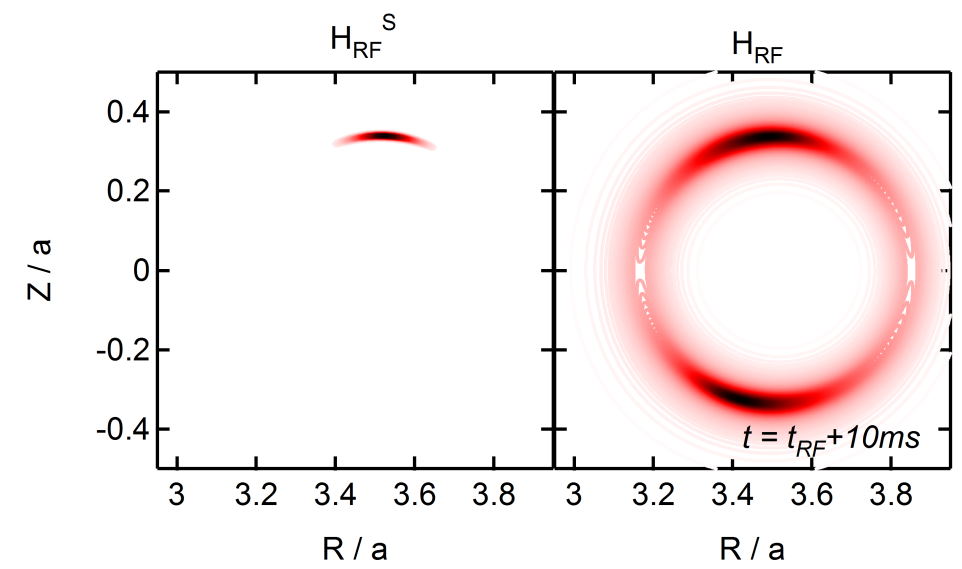

Figure 7. Normalized contour of the power density source $H_{R F}^{S}$ (left), and coupled power density $H_{R F} 10 \mathrm{~ms}$ after RF power is switched on (right).

The theoretical decay rate (using $S_{0} / S_{\text {res }}=2.17$ ) is therefore

$$
\left(-S_{0} \frac{d W}{d t}\right)^{t h} \approx 15.76 C_{\Omega}\left(\mu_{c}, \sigma\right)\left(\frac{P_{R F}}{P_{e q}}\right)^{1 / \sigma}
$$

The theoretical decay rates are obtained using $\delta_{H} \sim \delta_{R F}^{e f f}$ for computing $\mu_{c}\left(\mu_{c} \approx 0.2\right)$ and the analytical formula for $C_{\Omega}\left(\mu_{c}, \sigma\right)$. Note that the variation of $\mu_{c}$ during the island evolution and in between different cases (see paragraph 3.2.4) does not impact significantly the value of $\mathcal{F}_{\Omega}$. The maximum standard deviation on $\left(-S_{0} d W / d t\right)^{t h}$ due to this variation of $\mu_{c}$ does exceed 0.05 (in absolute value). If we would consider the actual magnetic shear when RF is applied, which is lower than the equilibrium one (see figure 2), the change in the theoretical value would be more important, with an increase of $9 \%$ to $15 \%$ that moves the theoretical points to larger decay rates.

3.2.3. Plasma response to RF heating The island response to the localized heating at the O-point results from the local increase of the temperature, from the associated decrease of plasma resistivity, and from the growth of a filament of ohmic current opposing the island current. In order to diagnose the spatial and dynamical aspects of the plasma response, we define several quantities that are representative of these key aspects. The temperature increase is simply measured by computing the evolution of the radial profile at $\theta^{O}=\pi / 2$, i.e. crossing the O-point of the island, and rescaling it with the radial profile in the absence of RF heating:

$\frac{\Delta T^{O}}{T^{O}}(\sqrt{\psi}, t)=\left\{T\left(\sqrt{\psi}, \theta^{O}, t\right)-T^{n o R F}\left(\sqrt{\psi}, \theta^{O}, t\right)\right\} / T^{n o R F}\left(\sqrt{\psi}, \theta^{O}, t\right)$

For measuring the impact on the parallel electric field and Ohmic current density (approximated by their toroidal projection), we compute quantities that are relevant for the island drive, i.e. the $n \neq 0$ projection of these fields, normalized to the $n=0$ 
profile in the absence of RF heating:

$\frac{\Delta E_{\|}^{O}}{E_{\|}^{O}}(\sqrt{\psi}, t)=\left\{E_{\varphi}^{n \neq 0}\left(\sqrt{\psi}, \theta^{O}, t\right)-E_{\varphi}^{n \neq 0, n o R F}\left(\sqrt{\psi}, \theta^{O}, t\right)\right\} / E_{\varphi}^{n=0, n o R F}\left(\sqrt{\psi}, \theta^{O}, t\right)(25)$
$\frac{\Delta J_{\Omega}^{O}}{J_{\Omega}^{O}}(\sqrt{\psi}, t)=\left\{J_{\Omega, \varphi}^{n \neq 0}\left(\sqrt{\psi}, \theta^{O}, t\right)-J_{\Omega, \varphi}^{n \neq 0, n o R F}\left(\sqrt{\psi}, \theta^{O}, t\right)\right\} / J_{\Omega, \varphi}^{n=0, n o R F}\left(\sqrt{\psi}, \theta^{O}, t\right)(26)$ where we use the relation $E_{\varphi}=\eta\left(J_{\varphi}-J_{C D, \varphi}\right)=\eta J_{\Omega, \varphi}$. In figure 8, we show the profiles of these quantities at $t-t_{R F}=0.025 \mathrm{~s}$, when the RF heat source and island decay rate are well established (see later). Because the temperature increase is very localized at the O-point for $\sigma=8$, it follows that the parallel electric field forms a deep well on a small radial scale, generating a strong localized Ohmic current. For $\sigma=1$, the temperature change diffuses to a broader area, the parallel electric field well is also broader and the ohmic current generated at the O-point is much less. The initial position of the O-point is at $\sqrt{\psi}^{O}=0.48$, indicated by a vertical dashed line. The perturbation that is visible around $\sqrt{\psi}=0.72$ is due to the $(3,1)$ sideband of the $(2,1)$ island. Note that for the quantities that are represented, the original and modified heat sources give similar results for $\sigma=1$. Outside the island separatrix, the heat diffusivity is larger for $\sigma=8$ than for $\sigma=1$ (this is not visible in the logarithmic scale used in figure 8 but it is clear on figures 3 and 6). This explains the smaller temperature gradient observed for $\sigma=8$ outside the island region compared to the case with $\sigma=1$.

In figure 9 , we show the time evolution of $\Delta T^{O} / T^{O}$ and $\Delta J_{\Omega}^{O} / J_{\Omega}^{O}$ at $\sqrt{\psi}^{O}=0.48$ where is the O-point. Due to the reduced diffusivity at $\sigma=8$, the temperature dynamics is faster at the RF heating application, but it also saturates earlier as the temperature gradient approaches its critical value where turbulence is activated. The saturation is lower for $\sigma=8$ than than for $\sigma=1$ because of the larger heat diffusivity that prevails outside the separatrix, as mentioned earlier. The contrast between $\sigma=1$ and $\sigma=8$ is particularly strong regarding the ohmic current density at the O-point, with a variation that is about one order of magnitude larger in stiff plasma conditions, but increases little with the RF power.

The perpendicular diffusivity inside the island, taken in between the O-point and the outer separatrix (at $\sqrt{\psi}=0.495$, where $\mathrm{RF}$ heating is restoring a temperature gradient) is shown in figure 10. As the injected power increases, the temperature gradient crosses the critical value where turbulent transport becomes large, and plasma diffusivity becomes comparable to its value without stiffness.

The plasma response to the $\mathrm{RF}$ heating shows therefore a strong sensitivity to the stiffness parameter, and this translates into the island decay rate.

3.2.4. Decay rate obtained in the simulations The dynamical aspect of the simulations is summarized in figure 11 for the case with the original background heat source, and in figure 12 for the modified heat source without background heating in the island region. The island decay rate $S_{0} d W / d t$ is obtained after taking into account the natural variation of the island size without RF heating, i.e. we take $W=W_{(2,1)}-W_{(2,1)}^{n o R F}+W_{(2,1)}^{n o R F}(t=$ 


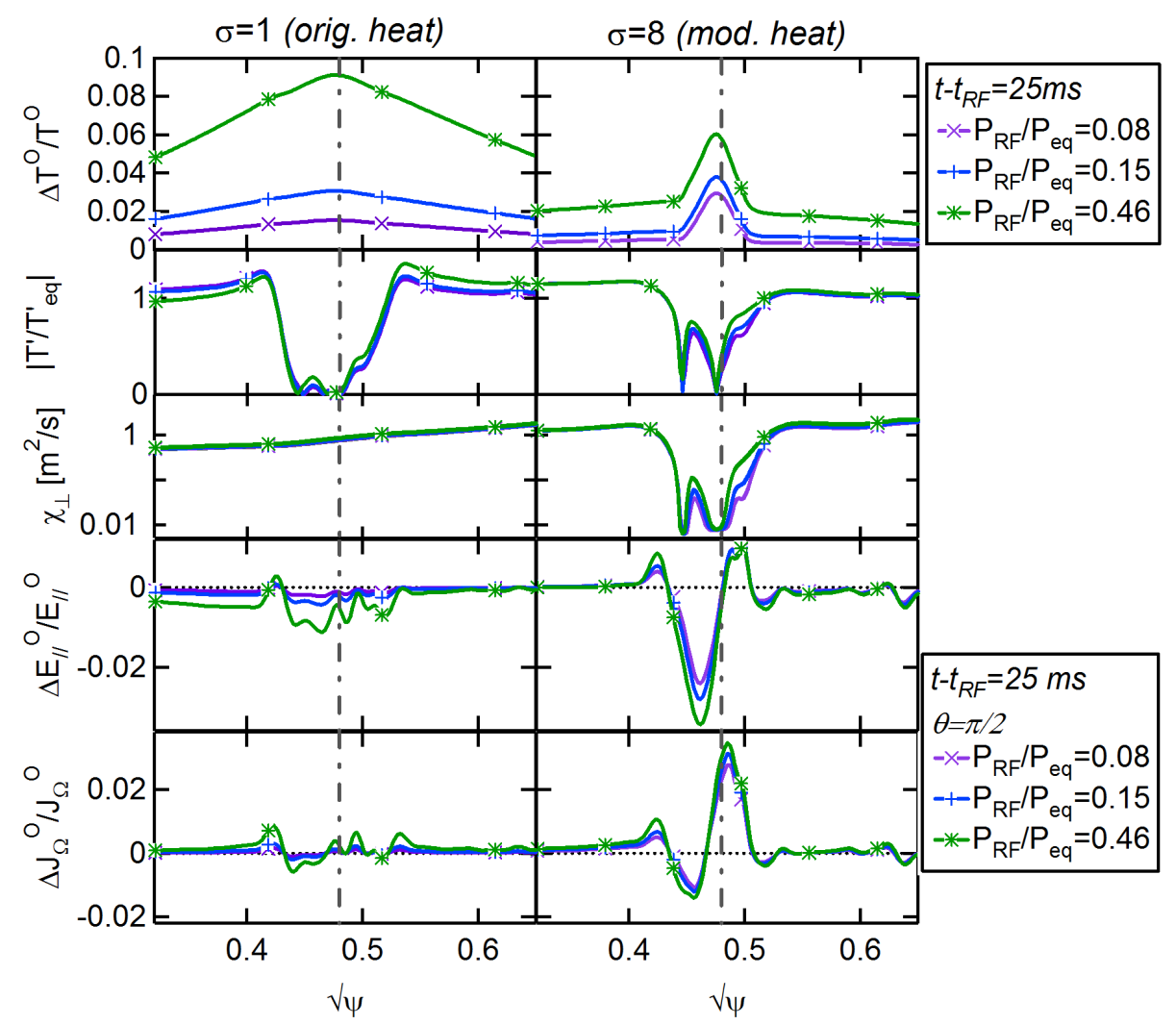

Figure 8. From top to bottom : radial profile (crossing the O-point) of $\Delta T^{O} / T^{O}$, normalized temperature gradient $\left|T^{\prime} / T_{e q}^{\prime}\right|$ (linear scale), perpendicular diffusivity (logarithmic scale), $\Delta E_{\|}^{O} / E_{\|}^{O}$ and $\Delta J_{\Omega}^{O} / J_{\Omega}^{O}$. The temperature stiffness is $\sigma=1$ in the left plots (with the original heat source) and $\sigma=8$ in the right plots (with the modified one). The vertical line is at the radial position of the O-point, $\sqrt{\psi}=0.48$.

$\left.t_{R F}\right)$. We have verified that the decay rate is then weakly dependent on the $\mathrm{RF}$ application time. For example, with $\sigma=1$ and the modified heat source where the time evolution of parameters is particularly important, the dynamics of $S_{0} d W / d t$ follows the same trajectory for an RF application at $t_{R F}=3.54 \mathrm{~s}$ and $t_{R F}=3.58 \mathrm{~s}$, with a difference in the decay rates after $25 \mathrm{~ms}$ that is of the order of $3 \%$. Finally, note that the decay rate is smoothed with a sliding time window of width $\Delta t=5 \mathrm{~ms}$.

The stiffness of the temperature profile has a clear effect, first, on the global plasma response to the addition of the RF power, as shown by the much weaker increase of the poloidal beta of the plasma for $\sigma=8\left(\beta_{p} \equiv 4 \int d V p /\left(\mu_{0} R_{0} I_{p}^{2}\right)\right.$ with $V$ the plasma volume, $p$ the pressure and $I_{p}$ the total plasma current). This results from the increased turbulence level as the heating source tends to increase the average temperature gradient above the critical gradient length. This has also an impact on the local response of the relative temperature fluctuation amplitude $\delta T / T$ (figure 11) and, as a consequence on the decay rate of the island, that is strongly reduced for the large values of $P_{R F} / P_{e q}$.

The important role of the background heat source in the case with stiff profiles and 


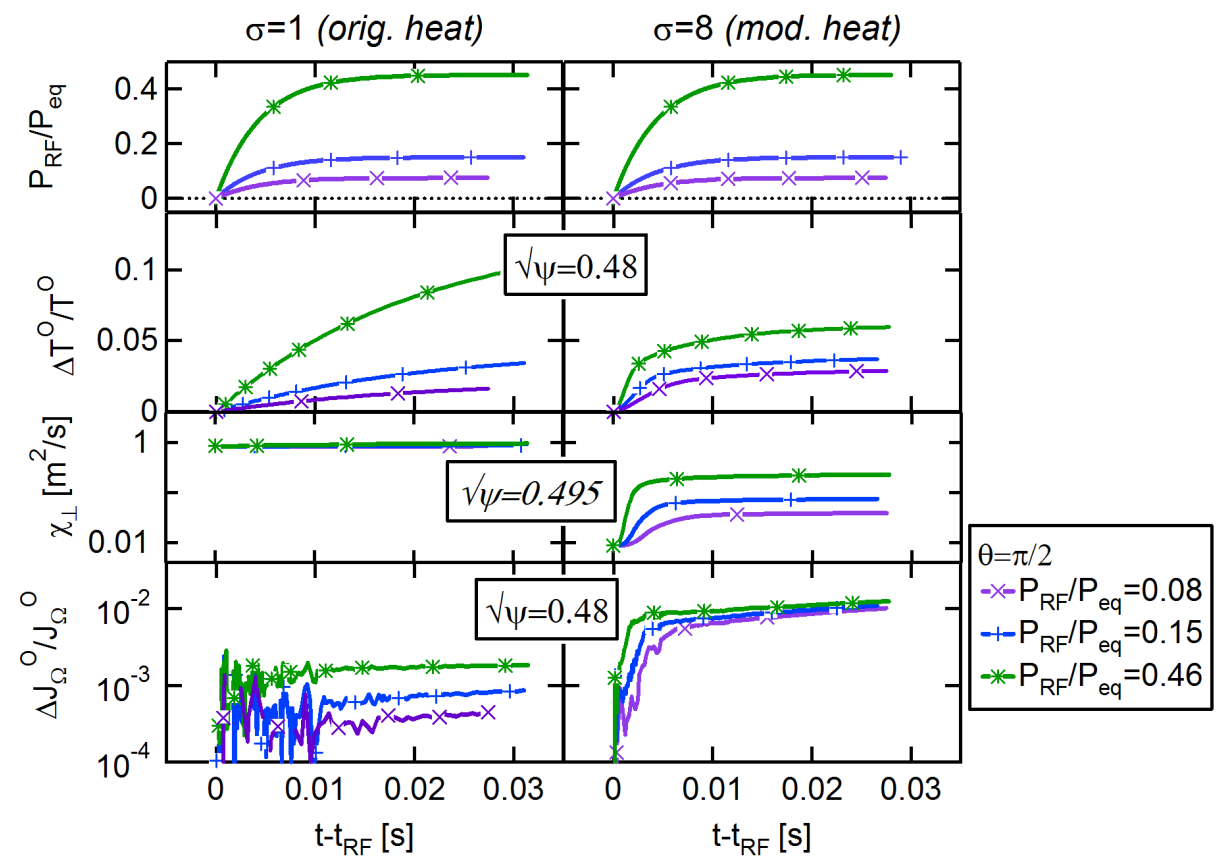

Figure 9. From top to bottom : time evolution of $P_{R F} / P_{e q}, \Delta T^{O} / T^{O}$, perpendicular diffusivity (at $\sqrt{\psi}=0.495$ ) and $\Delta J_{\Omega}^{O} / J_{\Omega}^{O}$ at the island O-point. The stiffness is $\sigma=1$ in the left plots (with the original heat source) and $\sigma=8$ in the right plots (with the modified one).

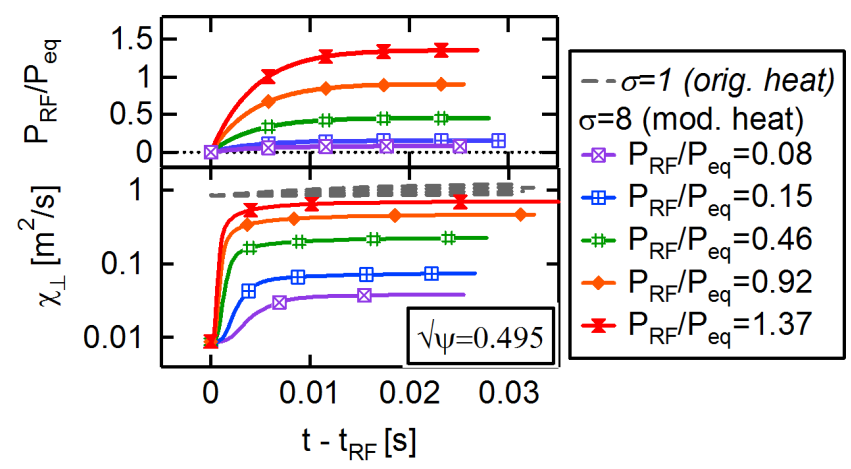

Figure 10. Time evolution of perpendicular diffusivity at $\sqrt{\psi}=0.495$

low ratio $P_{R F} / P_{e q}$ is illustrated in figure 13 . While the decay rate is moderate in the case where the residual background heat source has maintained a temperature gradient inside the island, it is much larger (and overcomes the $\sigma=1$ case) when the island region has an initial flat temperature profile. This is due to a level of turbulent transport that is already significant when the background heat source is present, as shown in the figure. The favorable effect of profile stiffness at reduced RF power can therefore vanish if other heating sources having no particular localization at the O-point of the island are at a level that can compete with the actuator of the control. In experimental conditions, this means that if a bump in the electron temperature is visible at the O-point before the 

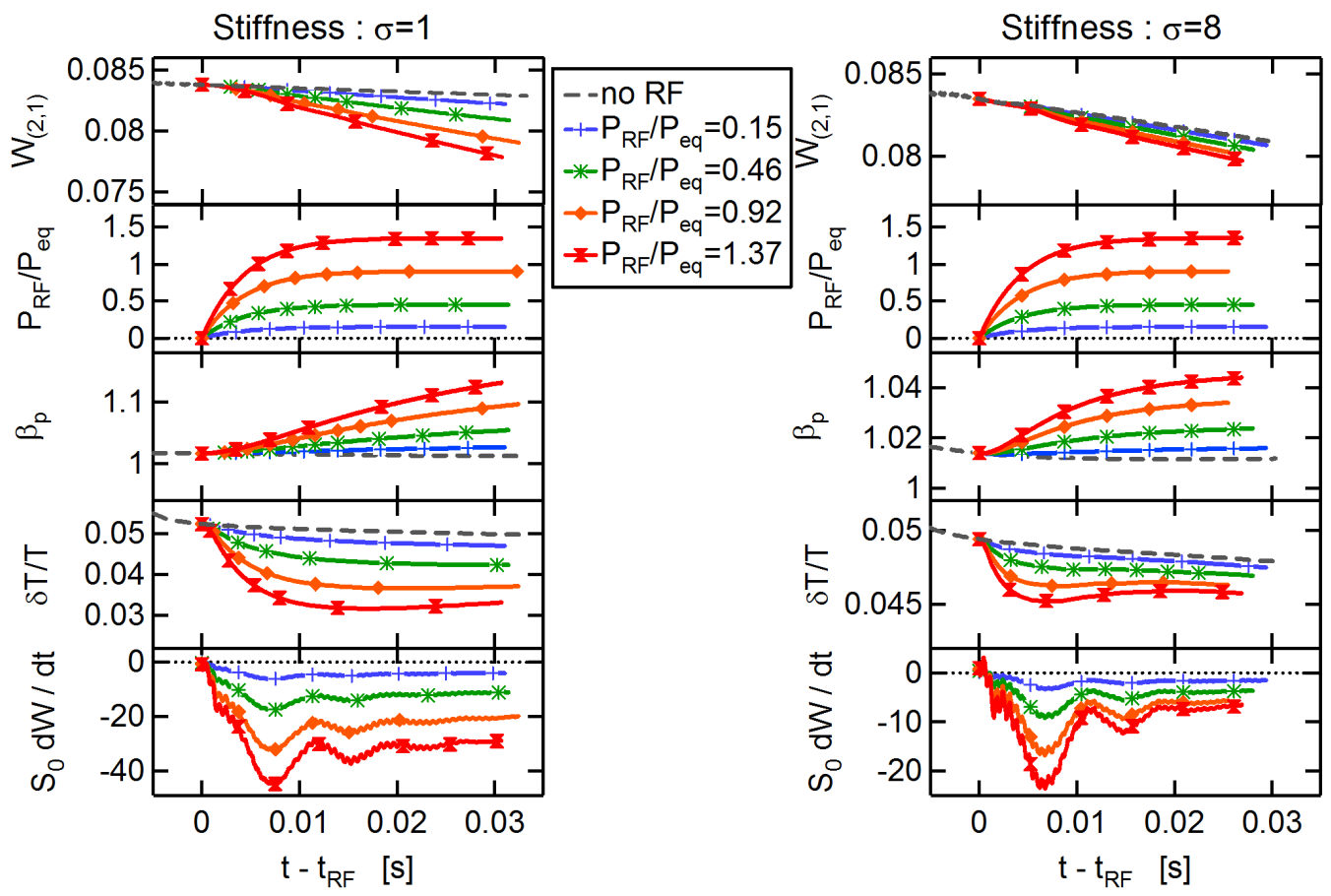

Figure 11. Island stabilization by localized RF heating for the original background heat source and $\sigma=1$ (left plots), $\sigma=8$ (right plots). From top to bottom: dynamics of the island width, $P_{R F} / P_{e q}$, poloidal beta of the plasma, relative temperature perturbation $\delta T / T$, and decay rate of the island normalized to the central Lundquist number.
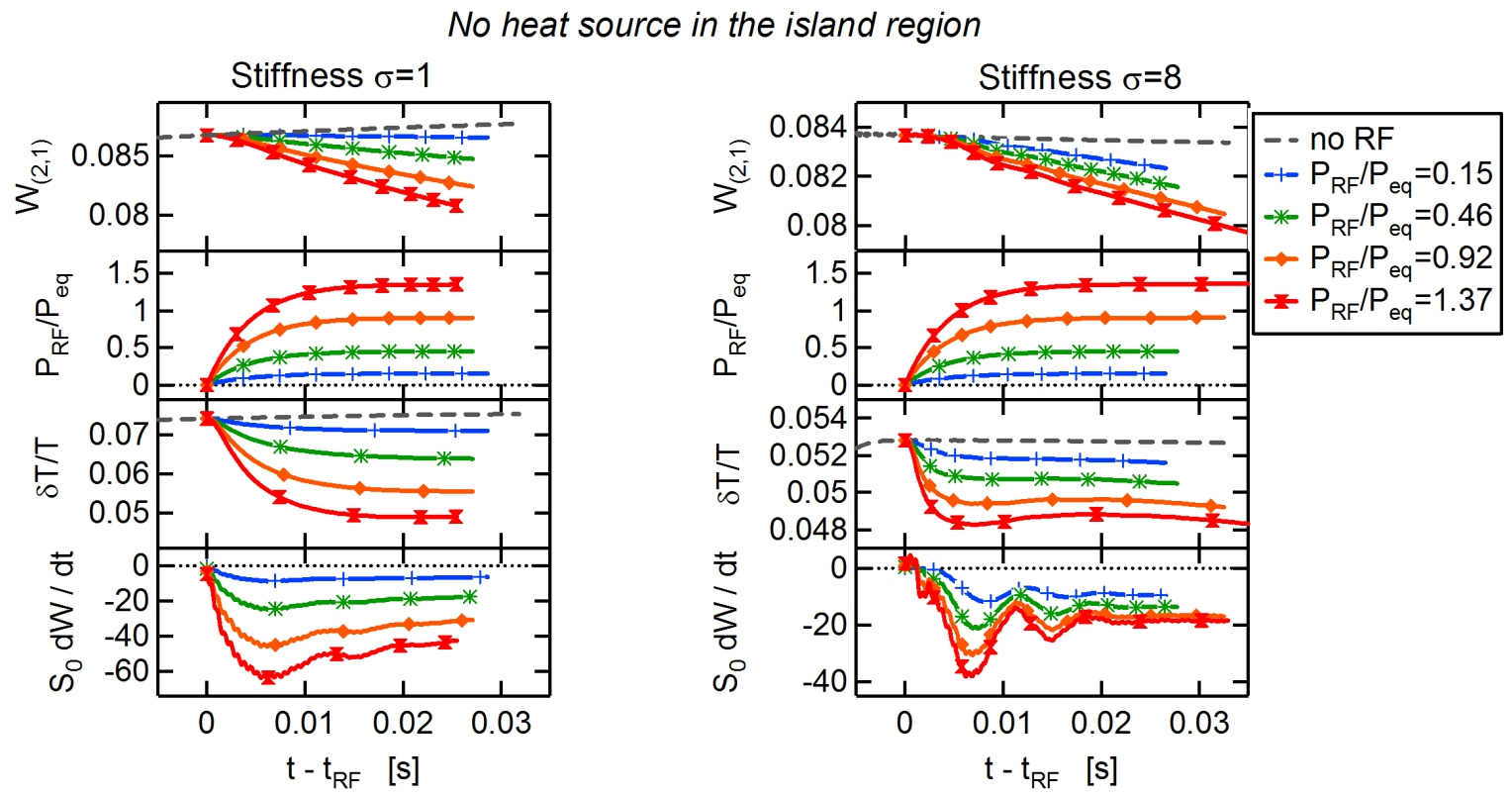

Figure 12. From top to bottom: dynamics of the island width, $P_{R F} / P_{e q}$, relative temperature perturbation $\delta T / T$, and decay rate of the island normalized to the central Lundquist number, for the case without background heating in the island region. 
RF application, then the efficiency of RF heating at the O-point will be weaker than expected. However, if the background heating in the island region reduces the impact of the control tool, it participates in the island reduction. This contribution can be evaluated using the formula for the theoretical decay rate, equation 23 , with $\mu_{c}=1$ and the power ratio $P_{e q}^{i s l} / P_{e q} \approx 0.31$. In principle, the contribution from this heat source that is one dimensional should be lower than the one from a O-point localized one as derived theoretically for $a \Delta_{\Omega}^{\prime}$, but as we will see later (section 3.2.5), the efficiency loss is already weak for a peaked heat source of width $\mu_{c}=0.2$, and we expect it to be even lower for a broad source at $\mu_{c}=1$. It appears therefore legitimate to identify the contribution from the background heating with $a \Delta_{\Omega}^{\prime}\left(P_{e q}^{i s l}\right)$. We obtain the following contributions:

$$
\begin{array}{rlrl}
\left(-S_{0} \frac{d W}{d t}\right)_{i s l}^{t h} & \approx 2.7 & \text { for } \sigma=1 \\
& \approx 11.0 \quad \text { for } \sigma=8
\end{array}
$$

The important stabilizing effect for $\sigma=8$ is due to its dependence as $\left(P_{e q}^{i s l} / P_{e q}\right)^{1 / \sigma}$, and it is likely producing the island decay seen in figure 2 , and its increase when removing the background heat source in figure 5 . The evaluation of the decay rate due to the residual heat source from the simulations at $\sigma=8$ gives actually a value of about 15 , consistent with the above estimate (see figure 13, right). It is easily understood that for $\sigma=8$, where the theoretical decay rate in the absence of background heating is about 10 for $P_{R F} / P_{e q}=0.15$, an offset of comparable amplitude, as computed above, severely reduces the impact of the controller. It is worth noting that, since $P_{e q}^{i s l}$ is (to leading order) proportional to the island width, we have here a potentially important mechanism, scaling as $W^{1 / \sigma}$, contributing to the island saturation.

The comparison of cases at $\sigma=1$ and $\sigma=8$ at the lowest RF power $\left(P_{R F} / P_{e q}=\right.$ 0.08 ), which is not shown in figures 11 and 12 for clarity, is detailed in figure 14. At this low ratio of $P_{R F} / P_{e q}$, the effect of transport stiffness is particularly important because the temperature gradient produced by RF heating inside the island remains well below the turbulence threshold, so that the effective diffusivity remains much lower than for $\sigma=1$ (see also figure 10).

The appropriate time when the decay rate is to be measured is set at $t-t_{R F}=$ $25 \pm 5 \mathrm{~ms}$, when the heating power has reached its nominal value and oscillations of the decay rate are stabilized. This time is much longer than the thermal equilibration time inside the island $\tau_{\chi}^{w}=w^{2} / \chi_{\perp} \approx 4 m s$. The simulation and theoretical results are displayed in figure 15 for the two stiffness parameters separately, and the most relevant cases (original heat source for $\sigma=1$ and modified one for $\sigma=8$ ) are compared in figure 16. For the theoretical results, we have included the computation using the actual magnetic shear (at the RF application), that gives a larger decay rate. The breaking of the linear dependence with respect to $P_{R F}$ when $\sigma>1$ is recovered, as well as the crossing of the decay rates at low $P_{R F} / P_{e q}$ (see figure 16).

The contributions of the background and RF heating cannot be treated additively in the stabilization term $a \Delta_{\Omega}^{\prime}$ because of their different radial extent (see 


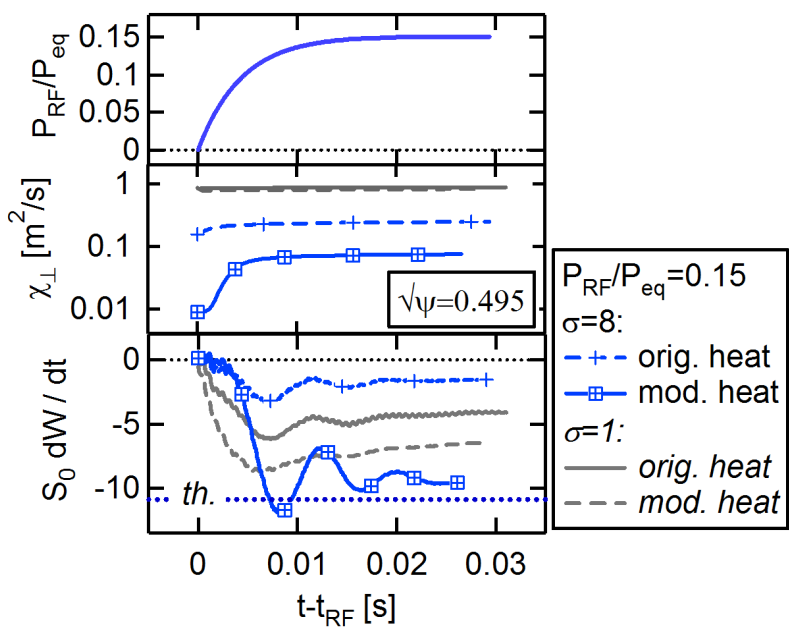

Effect of residual heat source

(no RF, $\sigma=8$ )

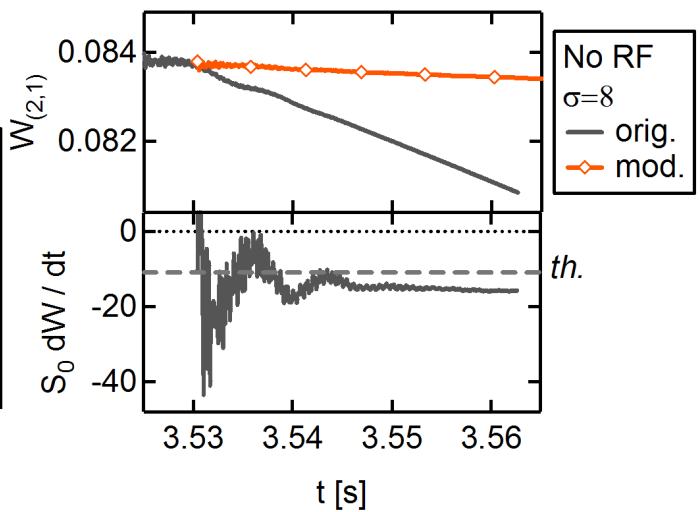

Figure 13. Role of the residual heat source on the RF control capability (left) and on the saturation without RF (right). Left : Injected RF power (top plot), perpendicular diffusivity inside the island at $\sqrt{\psi}=0.495$ (middle plot), and decay rate (bottom plot) for $P_{R F} / P_{e q}=0.15$ and $\sigma=8$, for the original heat source and the modified one without background heating in the island region. The case $\sigma=1$ is also indicated for comparison. Right : island width (top) and decay rate (bottom) for $\sigma=8$ without RF heating with the original $\left(P_{e q}^{i s l} / P_{e q} \approx 0.31\right)$ and modified $\left(P_{e q}^{i s l} / P_{e q} \approx 0\right)$ heat sources (the theoretical evaluation is indicated with a dashed line).

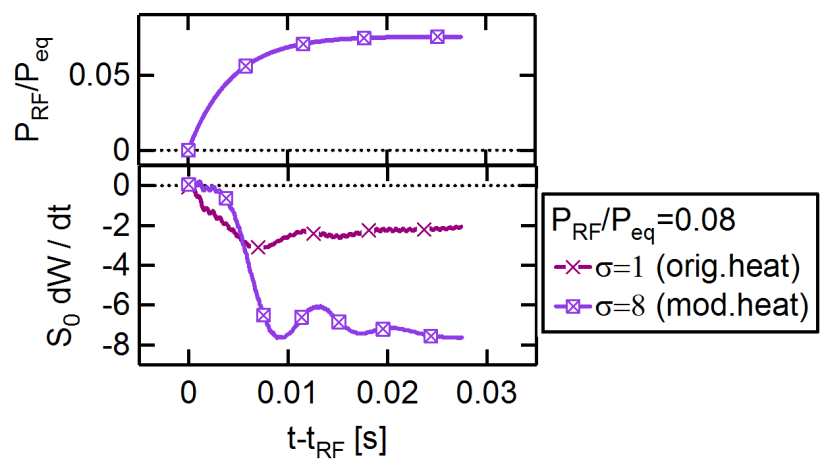

Figure 14. Injected RF power (top) and island decay rate (bottom) for $P_{R F} / P_{e q}=$ 0.08: comparison between $\sigma=1$ and $\sigma=8$.

[Maget et al., 2018]), but a satisfactory fit of the effective island decay rate due to the $\mathrm{RF}$ heating can be obtained by the following replacement:

$$
\left(\frac{P_{R F}}{P_{e q}}\right)^{1 / \sigma} \rightarrow\left(\frac{P_{R F}+\alpha P_{e q}^{i s l}}{P_{e q}}\right)^{1 / \sigma}-\left(\frac{\alpha P_{e q}^{i s l}}{P_{e q}}\right)^{1 / \sigma}
$$

with $\alpha=0.1$, a value that is expected to depend on the width of the RF deposition (see figure 15). The effective contribution of heating can then be schematically inserted in the Rutherford equation as :

$$
I_{1} \tau_{R} \partial_{t} W=a \Delta^{\prime}+a \Delta_{\Omega}^{\prime}\left(P_{R F}+\alpha P_{e q}^{i s l}\right)-a \Delta_{\Omega}^{\prime}\left(\alpha P_{e q}^{i s l}\right)+a \Delta_{\Omega}^{\prime}\left(P_{e q}^{i s l}\right)
$$




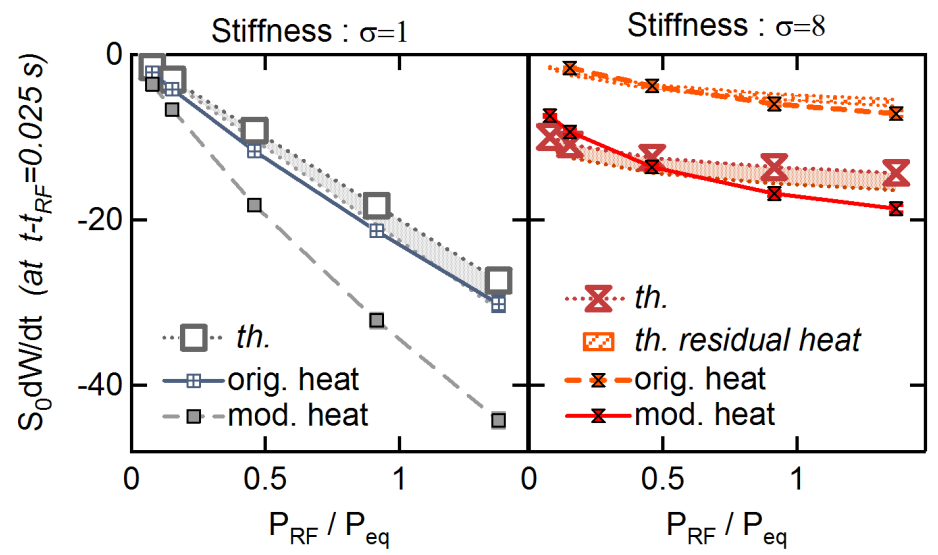

Figure 15. Decay rate of the island at $t-t_{R F}=0.025 \mathrm{~s}$ as a function of $P_{R F} / P_{e q}$ for $\sigma=1$ (left) and $\sigma=8$ (right) with the original and modified heat sources. The theoretical effective decay rate when taking into account the residual heat source in the island is also indicated for $\sigma=8$ (see equation (29)).
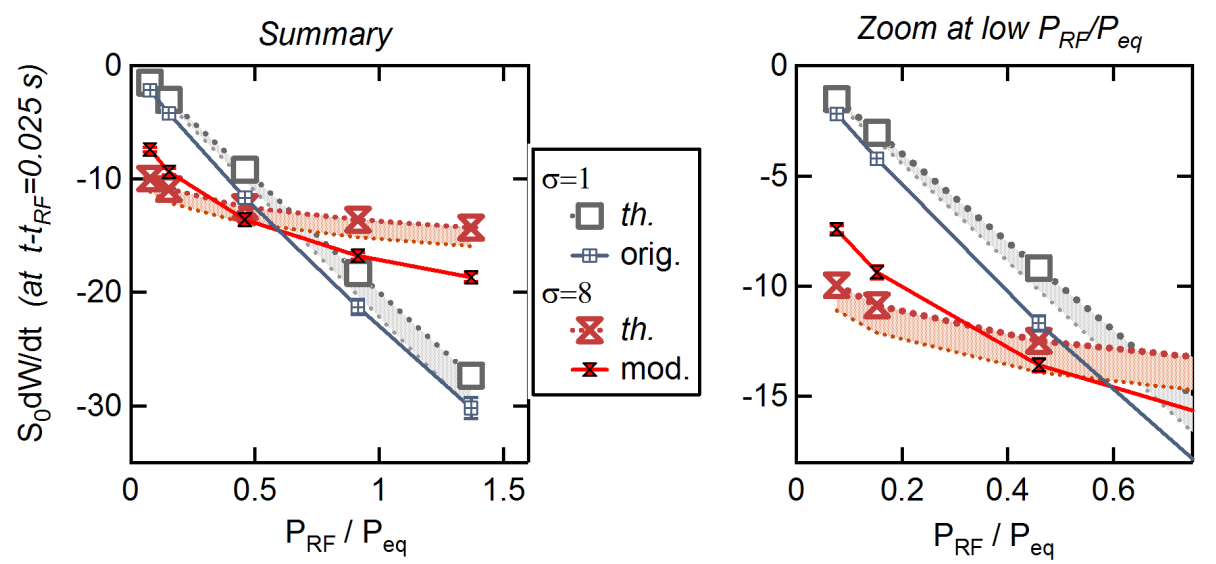

Figure 16. Decay rate of the island at $t-t_{R F}=0.025 \mathrm{~s}$ as a function of $P_{R F} / P_{e q}$ for the relevant heat sources (original heat source for $\sigma=1$ and modified heat source for $\sigma=8)$. Right plot: focus on low $P_{R F} / P_{e q}$ values.

3.2.5. One dimensional versus $3 D$ localized $R F$ heating Finally, we have investigated the efficiency of RF heating when the RF source is one dimensional. This is equivalent to the mean efficiency when the island is rotating and the RF heating crosses alternatively the O-point and the X-point of the island. This situation is not covered by the analytical model, but corresponds to a usual experimental set-up. Numerical simulations are shown in figure 17 for $\sigma=1$ (left plot) and $\sigma=8$ (right plot). In terms of stabilization efficiency, we find that the $1 \mathrm{D}$ RF heat source gives an island decay rate of about $74 \%$ of its continuous O-point value for $\sigma=1$, and $81 \%$ for $\sigma=8$. We conclude that profile 

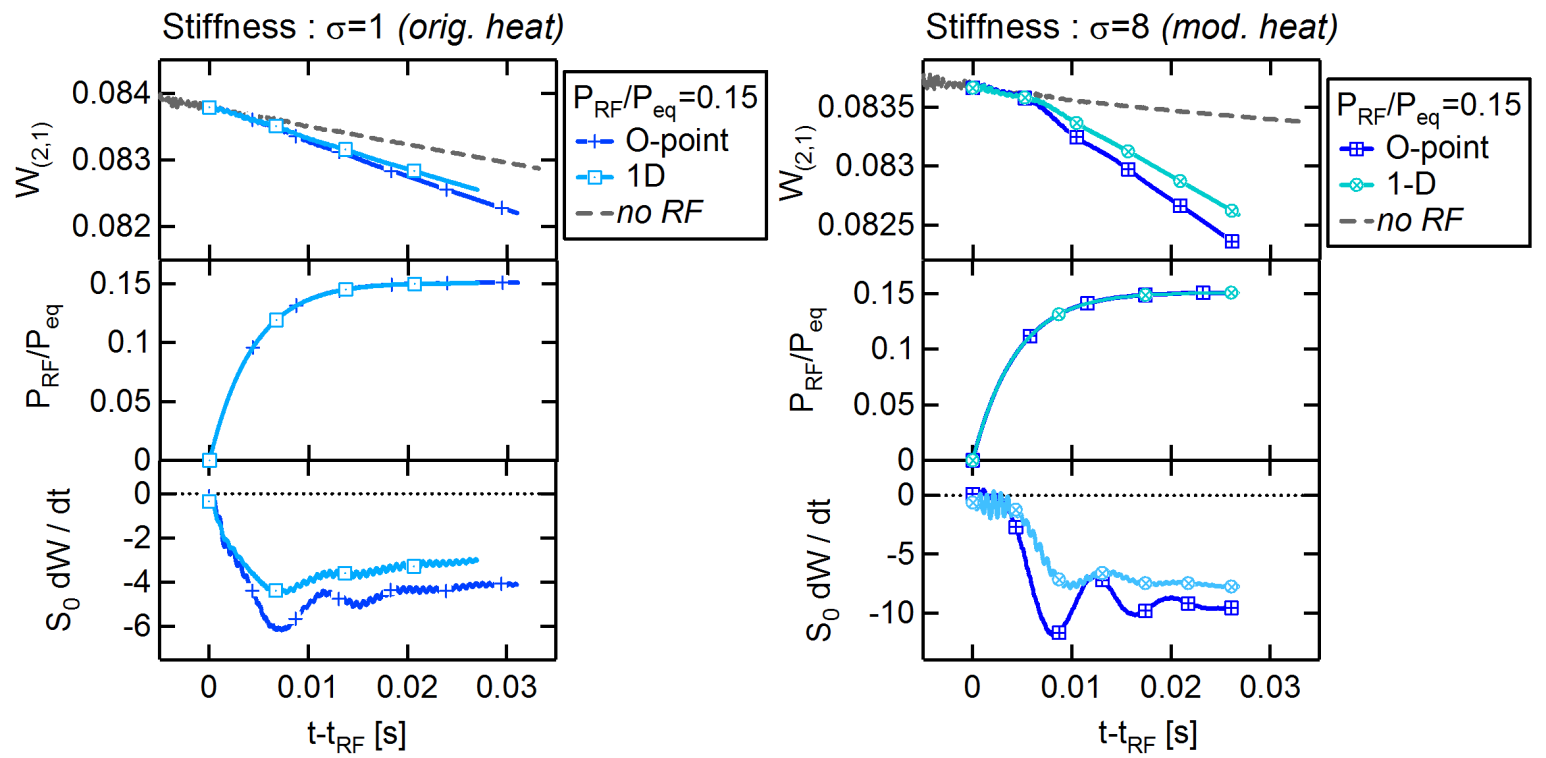

Figure 17. Comparison between O-point and 1-D (equivalent to continuous) RF application: island size (top), RF power (middle) and decay rate (bottom), for $\sigma=1$ (left, with the original heat source) and $\sigma=8$ (right, with the modified heat source).

stiffness does not modify significantly the stabilization potential of a non modulated RF source. It appears also than a continuous RF deposition does not bring a significant degradation of the stabilization efficiency.

\section{Conclusion}

In the present paper we have investigated numerically the stabilization efficiency of $\mathrm{RF}$ heating at the O-point of a magnetic island in a plasma where anomalous heat diffusivity is excited above a threshold in the temperature gradient, leading to the stiff temperature profiles that are observed in tokamaks. The settings where chosen so as to allow a comparison with an analytical derivation [Maget et al., 2018], and required a saturated island with a flat temperature inside the separatrix. This could be achieved at the expense of a large parallel conductivity, and for the case with a large stiffness parameter a modification of the background heat source was required. The main results can be summarized as follows:

(i) In agreement with the analytical model, we find that a large stiffness parameter leads to a stabilization efficiency by RF heating that is much larger than predicted without stiffness, in regimes where the ratio $P_{R F} / P_{e q}$ is small, as is generally the case in plasma experiments.

(ii) From the point of view of island control, the extreme sensitivity of the plasma response to the heat source in conditions of low turbulent transport has another important consequence related to the presence of residual heat sources (i.e. not 
due to the intentional deposition of RF waves) in the island region. These sources can indeed severely degrade the above mentioned enhancement if they are at a level that competes with the control heat source employed for the stabilization. This degradation is a direct consequence of the presence of a pre-existing bump in the temperature profile inside the island, that reduces the contrast brought by the actuator of the control, i.e. by the RF heat source. Therefore, a reliable signature of this possible loss of efficiency is the observation of such a bump before the RF application.

(iii) From the the point of view of island saturation, these residual heat sources have a favorable effect by reducing the island decay rate by a factor proportional to $W^{1 / \sigma}$ with $W$ the island width. Note that the analytical model of [Maget et al., 2018] can be readily extended to the case of an increased radiation level inside the island (due to impurity accumulation for example), where it predicts a destabilizing effect increasing as $\left(P_{\text {rad }} / P_{e q}\right)^{1 / \sigma}$ with $P_{\text {rad }}$ the power radiated inside the island. The large profile stiffness results therefore in a large sensitivity of the island saturation on both heat sources (stabilizing) and losses (destabilizing).

(iv) A Rutherford equation covering both the background and the RF heating is proposed (equation 30).

(v) The degradation of the stabilization efficiency when the heat source is 1D (i.e. continuous) instead of being focused at the O-point is comparable and moderate for both stiff and non-stiff temperature profiles.

The heating contribution to island stabilization by an RF source could therefore be significantly different from the theoretical expectation based on a uniform diffusivity model, i.e. where profile stiffness is not taken into account. This certainly applies to present experiments, with plasma parameters that are comparable to the one used in the present investigation. In ITER, the ratio $P_{R F} / P_{e q}$ should be relatively low (around $15 \%$ ), and the impact of a stiff transport rule should greatly enhance the role of $\mathrm{RF}$ heating compared to previous expectations, but in absolute value, this contribution is expected to be small compared to that of current drive [Lazzari and Westerhof, 2009, Lazzari and Westerhof, 2010]. The stabilizing contribution coming from the background heat source in the island region might provide, however, a significant stabilizing effect that remains to be evaluated.

\section{Acknowledgments}

This work has been carried out within the framework of the French Research Federation for Fusion Studies, and of the EUROfusion Consortium. It has received funding from the Euratom research and training programme 2014-2018 under grant agreement No 633053 for the project WP17-ENR-CEA-06. We benefited from HPC resources from GENCI (project 056348), from Marconi-Fusion (project NISMO) and from Aix-Marseille Université project Equip@Meso (ANR-10-EQPX-29-01) of the program 
"Investissements d'Avenir" supervised by the Agence Nationale pour la Recherche. This work is part of the project AMICI funded by the Agence Nationale pour la Recherche (ANR-14-CE32-0004-01). The views and opinions expressed herein do not necessarily reflect those of the European Commission.

\section{References}

[Bardòczi et al., 2017] Bardòczi, L., Carter, T. A., Haye, R. J. L., Rhodes, T. L., and McKee, G. R. (2017). Impact of neoclassical tearing modeturbulence multi-scale interaction in global confinement degradation and magnetic island stability. Physics of Plasmas, 24(12):122503, DOI: 10.1063/1.5004987, https://doi.org/10.1063/1.5004987.

[Bardòczi et al., 2016] Bardòczi, L., Rhodes, T. L., Carter, T. A., Bañòn Navarro, A., Peebles, W. A., Jenko, F., and McKee, G. (2016). Modulation of core turbulent density fluctuations by largescale neoclassical tearing mode islands in the diii-d tokamak. Phys. Rev. Lett., 116:215001, DOI: 10.1103/PhysRevLett.116.215001, https://link.aps.org/doi/10.1103/PhysRevLett. 116.215001.

[de Vries et al., 1997] de Vries, P. C., Waidmann, G., Krmer-Flecken, A., Donn, A. J. H., and Schller, F. C. (1997). Temperature profile perturbations due to magnetic islands in TEXTOR. Plasma Physics and Controlled Fusion, 39(3):439, http://stacks.iop.org/0741-3335/39/i=3/a=008.

[DeBoo et al., 2012] DeBoo, J. C., Petty, C. C., White, A. E., Burrell, K. H., Doyle, E. J., Hillesheim, J. C., Holland, C., McKee, G. R., Rhodes, T. L., Schmitz, L., Smith, S. P., Wang, G., and Zeng, L. (2012). Electron profile stiffness and critical gradient studies. Physics of Plasmas, 19(8):082518, DOI: $10.1063 / 1.4750061$, http://dx.doi.org/10.1063/1.4750061.

[Dimits et al., 2000] Dimits, A. M., Bateman, G., Beer, M. A., Cohen, B. I., Dorland, W., Hammett, G. W., Kim, C., Kinsey, J. E., Kotschenreuther, M., Kritz, A. H., Lao, L. L., Mandrekas, J., Nevins, W. M., Parker, S. E., Redd, A. J., Shumaker, D. E., Sydora, R., and Weiland, J. (2000). Comparisons and physics basis of tokamak transport models and turbulence simulations. Physics of Plasmas, 7(3):969-983, DOI: 10.1063/1.873896, http://dx.doi.org/10.1063/1.873896.

[Février et al., 2016] Février, O., Maget, P., Lütjens, H., Luciani, J. F., Decker, J., Giruzzi, G., Reich, M., Beyer, P., Lazzaro, E., Nowak, S., and the ASDEX Upgrade team (2016). First principles fluid modelling of magnetic island stabilization by electron cyclotron current drive (ECCD). Plasma Physics and Controlled Fusion, 58(4):045015, http://stacks.iop.org/0741-3335/58/i=4/a= 045015.

[Février et al., 2018] Février, O., Nicolas, T., Maget, P., Ahn, J.-H., Garbet, X., and Lütjens, H. (2018). Non-linear MHD simulations of sawteeth and their control by current and power depositions, https://hal.archives-ouvertes.fr/hal-01707731. working paper or preprint.

[Fitzpatrick, 1995] Fitzpatrick, R. (1995). Helical temperature perturbations associated with tearing modes in tokamak plasmas. Physics of Plasmas, 2(3):825-838, DOI: 10.1063/1.871434, http: //link.aip.org/link/?PHP/2/825/1.

[Fitzpatrick, 2017] Fitzpatrick, R. (2017). Effect of nonlinear energy transport on neoclassical tearing mode stability in tokamak plasmas. Physics of Plasmas, 24(5):052504, DOI: 10.1063/1.4982610, http://dx.doi.org/10.1063/1.4982610.

[Furth et al., 1963] Furth, H. P., Killen, J., and Rosenbluth, M. (1963). Finite-resistivity instabilities of a sheet pinch. Physics of Fluids, 6(4):459-484.

[Garbet et al., 2004] Garbet, X., Mantica, P., Angioni, C., Asp, E., Baranov, Y., Bourdelle, C., Budny, R., Crisanti, F., Cordey, G., Garzotti, L., Kirneva, N., Hogeweij, D., Hoang, T., Imbeaux, F., Joffrin, E., Litaudon, X., Manini, A., McDonald, D. C., Nordman, H., Parail, V., Peeters, A., Ryter, F., Sozzi, C., Valovic, M., Tala, T., Thyagaraja, A., Voitsekhovitch, I., Weiland, J., Weisen, H., Zabolotsky, A., and the JET EFDA Contributors (2004). Physics of transport in tokamaks. 
Plasma Physics and Controlled Fusion, 46(12B):B557, http://stacks.iop.org/0741-3335/46/ $\mathrm{i}=12 \mathrm{~B} / \mathrm{a}=045$.

[Hegna and Callen, 1997] Hegna, C. C. and Callen, J. D. (1997). On the stabilization of neoclassical magnetohydrodynamic tearing modes using localized current drive or heating. Physics of Plasmas, 4(8):2940-2946, DOI: 10.1063/1.872426, http://link.aip.org/link/?PHP/4/2940/1.

[Hill et al., 2015] Hill, P., Hariri, F., and Ottaviani, M. (2015). The effect of magnetic islands on ion temperature gradient turbulence driven transport. Physics of Plasmas, 22(4):042308, DOI: 10.1063/1.4919031, https://doi.org/10.1063/1.4919031.

[Hornsby et al., 2010] Hornsby, W. A., Peeters, A. G., Snodin, A. P., Casson, F. J., Camenen, Y., Szepesi, G., Siccinio, M., and Poli, E. (2010). The nonlinear coupling between gyroradius scale turbulence and mesoscale magnetic islands in fusion plasmas. Physics of Plasmas, 17(9):092301, DOI: 10.1063/1.3467502, http://dx.doi.org/10.1063/1.3467502.

[Hornsby et al., 2011] Hornsby, W. A., Siccinio, M., Peeters, A. G., Poli, E., Snodin, A. P., Casson, F. J., Camenen, Y., and Szepesi, G. (2011). Interaction of turbulence with magnetic islands: effect on bootstrap current. Plasma Physics and Controlled Fusion, 53(5):054008, http://stacks .iop. org/0741-3335/53/i=5/a=054008.

[Ida et al., 2012] Ida, K., Kamiya, K., Isayama, A., and Sakamoto, Y. (2012). Reduction of Ion Thermal Diffusivity Inside a Magnetic Island in JT-60U Tokamak Plasma. Phys. Rev. Lett., 109:065001, DOI: 10.1103/PhysRevLett.109.065001, https://link.aps.org/doi/10.1103/ PhysRevLett.109.065001.

[Imbeaux et al., 2001] Imbeaux, F., Ryter, F., and Garbet, X. (2001). Modelling of ECH modulation experiments in ASDEX Upgrade with an empirical critical temperature gradient length transport model. Plasma Physics and Controlled Fusion, 43(11):1503, http://stacks.iop.org/ $0741-3335 / 43 / i=11 / a=306$.

[Inagaki et al., 2004] Inagaki, S., Tamura, N., Ida, K., Nagayama, Y., Kawahata, K., Sudo, S., Morisaki, T., Tanaka, K., and Tokuzawa, T. (2004). Observation of Reduced Heat Transport inside the Magnetic Island O Point in the Large Helical Device. Phys. Rev. Lett., 92:055002, DOI: 10.1103/PhysRevLett.92.055002, https://link.aps.org/doi/10.1103/PhysRevLett. 92.055002.

[Izacard et al., 2016] Izacard, O., Holland, C., James, S. D., and Brennan, D. P. (2016). Dynamics of ion temperature gradient turbulence and transport with a static magnetic island. Physics of Plasmas, 23(2):022304, DOI: 10.1063/1.4941704, http://dx.doi.org/10.1063/1.4941704.

[Kasparek et al., 2016] Kasparek, W., Doelman, N., Stober, J., Maraschek, M., Zohm, H., Monaco, F., Eixenberger, H., Klop, W., Wagner, D., Schubert, M., Schtz, H., Grnwald, G., Plaum, B., Munk, R., Schlter, K., and Team, A. U. (2016). NTM stabilization by alternating Opoint EC current drive using a high-power diplexer. Nuclear Fusion, 56(12):126001, http: //stacks.iop.org/0029-5515/56/i=12/a=126001.

[Kinsey et al., 2011] Kinsey, J., Staebler, G., Candy, J., Waltz, R., and Budny, R. (2011). ITER predictions using the GYRO verified and experimentally validated trapped gyro-Landau fluid transport model. Nuclear Fusion, 51(8):083001, http://stacks.iop.org/0029-5515/51/i=8/ $\mathrm{a}=083001$.

[Kurita et al., 1994] Kurita, G., Tuda, T., Azumi, M., Takizuka, T., and Takeda, T. (1994). Effect of local heating on the $\mathrm{m}=2$ tearing mode in a tokamak. Nuclear Fusion, 34(11):1497, http: //stacks.iop.org/0029-5515/34/i=11/a=I08.

[Lazzari and Westerhof, 2009] Lazzari, D. D. and Westerhof, E. (2009). On the merits of heating and current drive for tearing mode stabilization. Nuclear Fusion, 49(7):075002, http://stacks .iop. org $/ 0029-5515 / 49 / i=7 / a=075002$.

[Lazzari and Westerhof, 2010] Lazzari, D. D. and Westerhof, E. (2010). On the merits of heating and current drive for tearing mode stabilization. Nuclear Fusion, 50(7):079801, http://stacks.iop. org $/ 0029-5515 / 50 / i=7 / a=079801$.

[Lütjens et al., 1996] Lütjens, H., Bondeson, A., and Sauter, O. (1996). The CHEASE 
code for toroidal MHD equilibria. Computer Physics Communications, 97(3):219260, http://www.sciencedirect.com/science/article/B6TJ5-3VSGD5T-19/2/ e429c1260ed10db93fde0078b7cee3e6.

[Lütjens and Luciani, 2010] Lütjens, H. and Luciani, J.-F. (2010). XTOR-2F: A fully implicit Newton-Krylov solver applied to nonlinear 3D extended MHD in tokamaks. Journal of Computational Physics, 229(21):8130 - 8143, ISSN: 0021-9991, DOI: DOI: 10.1016/j.jcp.2010.07.013, http://www.sciencedirect.com/science/article/ B6WHY-50JPNF3-2/2/2787626de93153effa740cca124bcfe7.

[Lütjens et al., 2001] Lütjens, H., Luciani, J.-F., and Garbet, X. (2001). Curvature effects on the dynamics of tearing modes in tokamaks. Physics of Plasmas, 8(10):4267-4270, http://link. aip.org/link/?PHP/8/4267/1.

[Maget et al., 2016] Maget, P., Février, O., Garbet, X., Lütjens, H., Luciani, J.-F., and Marx, A. (2016). Extended magneto-hydro-dynamic model for neoclassical tearing mode computations. Nuclear Fusion, 56(8):086004, http://stacks.iop.org/0029-5515/56/i=8/a=086004.

[Maget et al., 2018] Maget, P., Widmer, F., Février, O., Garbet, X., and Lütjens, H. (2018). Stabilization of a magnetic island by localized heating in a tokamak with stiff temperature profile. Physics of Plasmas, 25(2):022514, DOI: 10.1063/1.5021759, https://doi.org/10. 1063/1.5021759.

[Mantica et al., 2009] Mantica, P., Strintzi, D., Tala, T., Giroud, C., Johnson, T., Leggate, H., Lerche, E., Loarer, T., Peeters, A. G., Salmi, A., Sharapov, S., Van Eester, D., de Vries, P. C., Zabeo, L., and Zastrow, K.-D. (2009). Experimental Study of the Ion Critical-Gradient Length and Stiffness Level and the Impact of Rotation in the JET Tokamak. Phys. Rev. Lett., 102:175002, DOI: 10.1103/PhysRevLett.102.175002, https://link.aps.org/doi/10.1103/PhysRevLett. 102.175002.

[Maraschek, 2012] Maraschek, M. (2012). Control of neoclassical tearing modes. Nuclear Fusion, 52(7):074007, http://stacks.iop.org/0029-5515/52/i=7/a=074007.

[Navarro et al., 2017] Navarro, A. B., Bardòczi, L., Carter, T. A., Jenko, F., and Rhodes, T. L. (2017). Effect of magnetic islands on profiles, flows, turbulence and transport in nonlinear gyrokinetic simulations. Plasma Physics and Controlled Fusion, 59(3):034004, http://stacks.iop.org/ $0741-3335 / 59 / i=3 / a=034004$.

[Press et al., 2007] Press, W. H., Teukolsky, S. A., Vetterling, W. T., and Flannery, B. P. (2007). Numerical Recipes 3rd Edition: The Art of Scientific Computing. Cambridge University Press, New York, NY, USA, 3 edition, ISBN: 0521880688, 9780521880688.

[Rebut et al., 1988] Rebut, P., Lallia, P., and Watkins, M. (1988). The critical temperature gradient model of plasma transport: applications to JET and future tokamaks. Plasma Physics and Controlled Nuclear Fusion Research, 2:191.

[Romanelli et al., 2004] Romanelli, M., Bourdelle, C., and Dorland, W. (2004). Effects of high density peaking and high collisionality on the stabilization of the electrostatic turbulence in the Frascati Tokamak Upgrade. Physics of Plasmas, 11(8):3845-3853, DOI: 10.1063/1.1766031, http://dx.doi.org/10.1063/1.1766031.

[Rutherford, 1973] Rutherford, P. H. (1973). Nonlinear growth of the tearing mode. Physics of Fluids, 16(11):1903-1908, DOI: 10.1063/1.1694232, http://link.aip.org/link/?PFL/16/1903/1.

[Urso et al., 2010] Urso, L., Fischer, R., Isayama, A., Team, A. U., and Team, J.-. (2010). Application of the Bayesian analysis to the modified Rutherford equation for NTM stabilization. Plasma Physics and Controlled Fusion, 52(5):055012, http://stacks.iop.org/0741-3335/52/i=5/a= 055012.

[Volpe et al., 2009] Volpe, F. A. G., Austin, M. E., Haye, R. J. L., Lohr, J., Prater, R., Strait, E. J., and Welander, A. S. (2009). Advanced techniques for neoclassical tearing mode control in DIII-D. Physics of Plasmas, 16(10):102502, 102502, DOI: 10.1063/1.3232325, http://link.aip.org/ link/?PHP/16/102502/1.

[Westerhof et al., 2007] Westerhof, E., Lazaros, A., Farshi, E., de Baar, M., de Bock, M., Classen, 
I., Jaspers, R., Hogeweij, G., Koslowski, H., Krmer-Flecken, A., Liang, Y., Cardozo, N. L., and Zimmermann, O. (2007). Tearing mode stabilization by electron cyclotron resonance heating demonstrated in the TEXTOR tokamak and the implication for ITER. Nuclear Fusion, 47(2):85, http://stacks.iop.org/0029-5515/47/i=2/a=003.

[Zarzoso et al., 2015] Zarzoso, D., Hornsby, W., Poli, E., Casson, F., Peeters, A., and Nasr, S. (2015). Impact of rotating magnetic islands on density profile flattening and turbulent transport. Nuclear Fusion, 55(11):113018, http://stacks .iop.org/0029-5515/55/i=11/a=113018. 